\title{
Article \\ Emotion and Wellness Profiles of Herbal Drinks Measured Using Different Questionnaire Designs
}

\author{
Pannapa Hanmontree ${ }^{1}$, Witoon Prinyawiwatkul ${ }^{2}$ (i) and Amporn Sae-Eaw ${ }^{1, *(1)}$ \\ 1 Department of Food Technology, Faculty of Technology, Khon Kaen University, Khon Kaen 40002, Thailand; \\ pannapa@kkumail.com \\ 2 School of Nutrition and Food Sciences, Louisiana State University, Agricultural Center, \\ Baton Rouge, LA 70803, USA; wprinya@lsu.edu \\ * Correspondence: sampor@kku.ac.th; Tel.: +66-43-362-131
}

check for updates

Citation: Hanmontree, P.; Prinyawiwatkul, W.; Sae-Eaw, A. Emotion and Wellness Profiles of Herbal Drinks Measured Using Different Questionnaire Designs. Foods 2022, 11, 348. https://doi.org/ $10.3390 /$ foods 11030348

Academic Editor: Han-Seok Seo

Received: 10 December 2021

Accepted: 22 January 2022

Published: 26 January 2022

Publisher's Note: MDPI stays neutral with regard to jurisdictional claims in published maps and institutional affiliations.

Copyright: (C) 2022 by the authors. Licensee MDPI, Basel, Switzerland. This article is an open access article distributed under the terms and conditions of the Creative Commons Attribution (CC BY) license (https:// creativecommons.org/licenses/by/ $4.0 /)$.

\begin{abstract}
The emotion and wellness profiles of herbal drinks were assessed using six different questionnaire designs. The questionnaire designs were constructed from two formats of questionnaire items, including words and sentences, and three types of measuring scales, including a rating scale (5-point intensity; 1 = 'not at all', 5 = 'extremely'), a checklist scale (check-all-that-apply, CATA), and a combination of CATA and rating scales (rate-all-that-apply, RATA; 5-point intensity; 1 = 'slightly', 5 = 'extremely'). The 39 emotional terms of the EsSense Profile ${ }^{\circledR}$ and the 45 wellness terms of the WellSense Profile ${ }^{\mathrm{TM}}$ were translated into Thai, then screened for relevance to herbal drinks. The seven positive emotional terms (active, energetic, good, happy, polite, satisfied, and warm), three negative emotional terms (bored, disgusted, and worried), and five wellness terms (comforted, healthy, invigorated, relaxed, and refreshed) were selected and included in the questionnaire. A central location test was performed to determine the emotion and wellness profiles of five herbal drinks: roselle (Hibiscus sabdariffa) drink, chrysanthemum (Chrysanthemum morifolium Ramat) drink, ginger (Zingiber officinale) drink, Jubliang (a mixture of eight herbs) drink, and Krachai Dam (Kaempferia parviflora) drink. For herbal drinks, measuring emotion and wellness with a questionnaire using full sentences did not show increased benefit over questionnaires using words alone. All three measuring methods-a rating scale, CATA, and RATA—produced similar emotion and wellness profiles. However, each method has different advantages and limitations, which researchers should carefully consider.
\end{abstract}

Keywords: emotion; wellness; herbal drink; product discrimination; questionnaire designs

\section{Introduction}

In the last decade, emotional responses have been more frequently used in consumer research and food-product development because the use of liking data alone may not effectively predict product success in the market. Collecting emotional responses that offer insight into consumer experience can provide additional useful information for product development [1]. Several findings have noted that emotions influence consumers' eating behavior and decision-making processes [2-4]. Understanding the emotions elicited by their products could be beneficial for food industries when considering packaging design, branding, and advertising [2].

Several methods for measuring emotions associated with foods have been developed and reported, such as EsSense Profile ${ }^{\circledR}$ [5], consumer-defined check-all-that-apply (CDCATA) [6], EmoSemio [7], and EmoSensory ${ }^{\circledR}$ Wheel [8]. Furthermore, the health and wellness perception of food products has become an important aspect of consumers' purchasing decisions. The WellSense Profile ${ }^{\mathrm{TM}}$, a questionnaire measuring wellness associated with foods from the consumer perspective, was recently developed [9] and was adapted in recent research to measure organic food-related wellbeing [10] in relation to the Krachai 
Dam drink [11]. Accordingly, the emotion and wellness responses of consumers should be considered in food-product development. Although the EsSense profile ${ }^{\circledR}$ and the WellSense Profile $^{\mathrm{TM}}$ methods have been widely used, the application of these methods in in countries with different cultures and languages should be appropriately performed. For this reason, cross-cultural studies of emotion elicited by food have gained attention [12-14]. However, research investigating the impact of questionnaire design and measurement scales on emotion and wellness profiles is scarce. In Thailand, methods for measuring emotional response elicited by food products have not been widely applied, so more research is required.

Herbs have been recognized as having health benefits [15]. Herbs and their extracts contain different bioactive compounds that can provide therapeutic effects, such as reducing cardiovascular problems, enhancing immune functions, and preventing cancer [16]. In Thailand, herbs have been used as medicine and food for a long time. Herbal drinks are becoming more popular, especially among health-conscious consumers, since these beverages are prepared with natural ingredients. The results obtained in a study by Jabeur et al. [17] highlighted the potential of Hibiscus sabdariffa L. (Hs), also known as roselle, as a source of bioactive and natural coloring ingredients destined for food and pharmaceutical industries. Roselle extracts showed antibacterial, antioxidant, anticholesterol, antidiabetic and antihypertensive effects, among others [18]. In recent years, the flowers of medicinal chrysanthemum (Chrysanthemum morifolium Ramat) were shown to act as common materials in functional and healthy tea or beverages due to their unique flavor, color, and health benefits, such as detoxification, improving liver function, decreasing inflammation, and improving eyesight [19]. Ginger (Zingiber officinale) rhizomes are commonly used in foods and beverages for their characteristic pungency and piquant flavor and have exhibited various pharmacological effects, such as antioxidant, anti-inflammatory, gastroprotective, antibacterial, and antidiabetic properties [20]. Jubliang is a mixture of eight herbs, namely Bombax ceiba L., Chrysanthemum morifolium, Imperata cylindrical (L.) P. Beauv., Lophatherum gracile Brogn., Nelumbo nucifera Gaertn, Oroxylum indicum L., Pragmites communis Trin, and Prunella vulgaris. Jubliang extract is a good source of water-soluble antioxidants, phenolic compounds, and antimutagens [21]. Kaempferia parviflora, or Krachai Dam (KD), is a Thai herb that belongs to the Zingiberaceae family. Its rhizomes have been reputed to have beneficial medicinal effects, owing to its major methoxyflavones, including anticancer, cardioprotective, neuroprotective, antioxidative, antimicrobial, and transdermal-permeable activity [22].

There are many kinds of commercial herbal drinks in Thailand, some of which are very popular; others are not popular, even though they have health-promotion benefits. There are many factors that have influence consumer perception and buying decisions. An important factor is the emotion and wellness responses of consumers. Additionally, different questionnaire designs may influence the results of emotion and wellness assessments. We hypothesized that using a questionnaire designed with full sentences and a rating-scale measurement method would have a greater influence on emotion and wellness profiles than that with words and a check-all-that-apply (CATA) measurement method. Therefore, in this research, we aimed to examine and compare the emotion and wellness responses associated with herbal drinks using questionnaires constructed according to two formats-words vs. full sentences - and three types of measurement methods, including a rating scale, a checklist scale (CATA), and a combination of the two scales (i.e., RATA).

\section{Materials and Methods}

\subsection{Herbal Drink Samples}

Five herbal drink samples commercially available in Thailand were used, including roselle drink, chrysanthemum drink, ginger drink, Jubliang drink, and Krachai Dam drink (Table 1). Roselle drink, chrysanthemum drink, Jubliang drink, and Krachai Dam drink were ready-to-drink products. After purchased, they were kept at $4{ }^{\circ} \mathrm{C}$ until used for consumer testing. Samples were presented to the participants in random 3-digit labelled cups at approximately $10^{\circ} \mathrm{C}$, except ginger drink, which was served at approximately 
$70{ }^{\circ} \mathrm{C}$. The ginger drink was prepared immediately prior to sampling by adding $18 \mathrm{~g}$ of instant ginger powder to $150 \mathrm{~mL}$ of hot water and kept in an insulated bottle.

Table 1. The description of five herbal drink samples.

\begin{tabular}{|c|c|c|}
\hline Herbal Drinks & Main Ingredients & Serving Condition/Temperature \\
\hline $\begin{array}{l}\text { Roselle drink } \\
\text { Ready to drink, } \\
200 \mathrm{~mL} / \text { box }\end{array}$ & Roselle extract $94 \%$, sucrose $6 \%$ & Cold $/ 10^{\circ} \mathrm{C}$ \\
\hline $\begin{array}{l}\text { Chrysanthemum drink } \\
\text { Ready to drink, } \\
350 \mathrm{~mL} / \text { bottle }\end{array}$ & Chrysanthemum extract $90.00 \%$, fructose syrup $4.00 \%$, sucrose $4.00 \%$ & Cold $/ 10^{\circ} \mathrm{C}$ \\
\hline $\begin{array}{c}\text { Ginger drink } \\
\text { Instant powder }{ }^{1} \\
18 \mathrm{~g} / \text { sachet }^{2}\end{array}$ & $\begin{array}{c}\text { Ginger extract } 8.03 \% \text {, sugar } 10.47 \% \text {, vitamin B1 } 0.0003 \% \text {, vitamin B2 } \\
0.0003 \% \text {, vitamin B6 } 0.0009 \%\end{array}$ & $\mathrm{Hot} / 70^{\circ} \mathrm{C}$ \\
\hline $\begin{array}{l}\text { Jubliang drink } \\
\text { Ready to drink, } \\
300 \text { mL/box }\end{array}$ & Jubliang extract $94.68 \%$, fructose $4.0 \%$, sugar $1.0 \%$ & Cold $/ 10^{\circ} \mathrm{C}$ \\
\hline $\begin{array}{l}\text { Krachai Dam drink } \\
\text { Ready to drink, } \\
90 \mathrm{~mL} / \text { bottle }\end{array}$ & $\begin{array}{c}\text { Krachai Dam extract } 20 \% \text {, fructose } 18 \% \text {, honey } 1.5 \% \text {, citric acid } 0.5 \% \text {, } \\
\text { vitamin C } 0.12 \% \text {, vitamin A } 0.012 \% \text {, vitamin B6 } 0.004 \%\end{array}$ & Cold $/ 10^{\circ} \mathrm{C}$ \\
\hline
\end{tabular}

${ }^{1}$ ginger drink was prepared by diluting $18 \mathrm{~g}$ of instant ginger powder in $150 \mathrm{~mL}$ of hot water.

\subsection{Screening of Emotion and Wellness Terms for Relevance to Herbal Drinks}

The research protocol concerned with the use of human subjects was approved by the Khon Kaen University Ethics Committee for Human Research (KKUEC), Thailand (approval code, HE611230). Emotion and wellness terms were selected from the EsSence Profile $^{\circledR}[5]$ and WellSense Profile ${ }^{\mathrm{TM}}$ tests [9]. All terms were translated into Thai by a group of experienced linguists $(n=3)$ and back-translated into English by another experienced group $(n=3)$ for meaning validation (Table 2$)$. Thai emotion and wellness terms relevant to five herbal drinks (Roselle drink, Chrysanthemum drink, Ginger drink, Jubliang drink, and Krachai Dam drink) were screened using online CATA questionnaires. Thai consumers ( $n=1678 ; 43.1 \%$ males and $56.9 \%$ females) who regularly consume herbal drinks participated voluntarily. Participants were asked to select the emotion and wellness terms that they associate with when thinking about herbal drinks that they had consumed. Terms with at least $20 \%$ frequency of use were recommended as a criterion $[2,5,23,24]$. However, terms with least $15 \%$ frequency of use were adopted for development of the EmoSensory ${ }^{\circledR}$ Wheel, while negative emotion terms with a lower frequency of use $(\leq 10 \%)$ were used to obtain a balance of positive and negative terms [8]. For our current research, terms with at least $18 \%$ frequency of use were used. First, the selected terms from all of five herbal drinks were collected. Then, the duplicated terms were removed before inclusion in the questionnaire. Based on the results, seven positive emotion terms (active, energetic, good, happy, polite, satisfied, and warm) and five wellness terms (comforted, healthy, invigorated, relaxed, and refreshed) were selected. To control the balance on both of positive and negative terms, three negative emotion terms (bored, disgusted, and worried) were added to the questionnaire (Table 3). Therefore, a total of 15 emotion and wellness terms were included in the questionnaire to measure the consumer responses elicited by the five herbal drinks.

\subsection{Testing the Test Designs for Measurement of Emotion and Wellness Responses Elicited by Herbal Drinks}

The proposed test designs for measurement of emotion and wellness responses were composed of 2 factors. Factor 1 was composed of two formats of questionnaire items, including words and full sentences (see the attached questionnaire file). The sentence format was used to reduce ambiguity within the questionnaire because including a context increases understanding of the specified emotion and wellness state. The full sentences 
were created using selected emotion and wellness terms, in consultation with a focus group of consumers ( $n=29$, average age $=45.76 \pm 7.83$ years old) (adapted from [7]). Factor 2 was composed of three types of measuring scales, including a rating scale (5-point intensity; $1=$ 'not at all', 5 = 'extremely'), a checklist scale (CATA), and a combination (rate-all-thatapply, RATA; 5-point intensity; $1=$ 'slightly', 5 = 'extremely'). Thus, the questionnaire designs comprised six test forms (Table 4).

Table 2. Thai translation of emotion and wellness terms obtained from the EsSense Profile ${ }^{\circledR}$ and the WellSense Profile ${ }^{\mathrm{TM}}$.

\begin{tabular}{|c|c|c|c|c|c|}
\hline \multicolumn{6}{|c|}{ EsSense Profile ${ }^{\circledR}$ (39 terms) } \\
\hline Active & รู้สึกกระฉับกระเฉง & Glad & ยินดี/ดีใจ & Pleased & พึงพอใจ \\
\hline Adventurous & ชอบผจญภัย & Good & ดี & Polite & สุภาพ \\
\hline Affectionate & รักใคร่ & Good-natured & มีใจเมตตา & Quiet & เงียบ \\
\hline Aggressive & ก้าวร้าว & Guilty & รู้สึกผิด & Satisfied & พึงพอใจ \\
\hline Bored & เบื่อ & Happy & มีความสุข & Secure & ปลอดภัย \\
\hline Calm & สงบ & Interested & รู้สึกสนใจ & Steady & คงเส้นคงวา \\
\hline Daring & กล้าหาญ & Joyful & ร่าเริง & Tame & จืดชืด/ไม่น่าสนใจ \\
\hline Disgusted & น่ารังเกียจ/ขยะแขยง & Loving & รู้สึกรัก & Tender & นุ่มนวล \\
\hline Eager & กระตือรือร้น & Merry & รู้สึกคครึกครื้น & Understanding & มีความเข้าอกเข้าใจ \\
\hline Energetic & รู้สึกมีพลัง & Mild & อ่อนโยน & Warm & อบอุ่น \\
\hline Enthusiastic & กระตือรือร้น & Nostalgic & ระลึกถึงความหลัง & Whole & รู้สึกสมบูรณ์ครบถ้วน \\
\hline Free & เป็นอิสระ & Peaceful & สงบ & Wild & คึกคะนอง \\
\hline Friendly & เป็นมิตร & Pleasant & รู้สึกกเพลิดเพลิน & Worried & รู้สึกกังวล \\
\hline \multicolumn{6}{|c|}{ WellSense Profile ${ }^{\mathrm{TM}}$ (45 terms) } \\
\hline Accepted & ได้รับการยอมรับ & Energetic & เต็มไปด้วยพลัง & Resilient & ยืดหยุ่น \\
\hline Accomplished & บรรลุเป้าหมาย & Fatigued & เหนื่อยล้า & Rested & ได้พักผ่อน \\
\hline Active & รู้สึกกระฉับกระเฉง & Focused & ตั้งใจจดจ่อ & Sad & เศร้า \\
\hline Affectionate & รักใคร่ & Friendly & เป็นมิตร & Satisfied & พึงพอใจ \\
\hline Alert & ตื่นตัว & Fulfilled & รู้สึกได้รับการเติมเต็ม & Secure & ปลอดภัย \\
\hline Approachable & เข้าถึงได้ & Grateful & กตัญญรู้คุณ & Sociable & ชอบเข้าสังคม \\
\hline Attentive & เอาใจใส่ & Happy & มีความสุข & Stimulated & ถูกกระตุ้น \\
\hline Calm & สงบ & Healthy & มีสุขภาพดี & Stressed & เครียด \\
\hline Comforted & รู้สึกสบาย & Invigorated & มีชีวิตชีวา & Supported & ได้รับการสนับสนุน \\
\hline Compassionate & เห็นอกเห็นใจ & Joyful & ร่าเริง & Unfulfilled & ยังไม่ได้รับการเติมเต็ม \\
\hline Concerned for others & มีความห่วงใยต่อผู้อื่น & Lonely & เหงา & Uninspired & ไม่มีแรงบันดาลใจ \\
\hline Connected & ผูกพัน & Loved & รู้สึกได้รับความรัก & Uninterested & ไม่สนใจ \\
\hline Creative & สร้างสรรค์ & Peaceful & สงบ & Tense & เครียด \\
\hline Curious & อยากรู้อยากเห็น & Refreshed & สดชื่น & Tired & เหนื่อยล้า \\
\hline Disconnected & ไม่ผูกพัน & Relaxed & รู้สึกผ่อนคลาย & Whole & รู้สึกสมบูรณ์ครบถ้วน \\
\hline
\end{tabular}

The impact of the test designs (Test 1 to Test 6) was evaluated between 15 and 30 June 2018. Consumers were recruited by an accidental sampling method. According to Meilgaard et al. [25], 50-300 responses should be collected per location for the central location test (CLT). In this study, about 200 Thai consumers were recruited for each test design (a total of 1252; 49.3\% males and 50.7\% females) from 4 regions of Thailand (Table 4), with almost equal proportions of four age groups participating in the CLT. To be selected, consumers were required to be familiar with and frequently consumers of herbal drinks. Each consumer was randomly assigned to one of the six test designs. Five herbal drinks 
were coded with random 3-digit numbers. Each herbal drink sample $(20 \mathrm{~mL})$ was poured into a clear plastic cup and served under the corresponding condition/temperature described in Table 1. All five samples were served in a balanced order. All consumers received five herbal drink samples for tasting, along with water to cleanse their palates between samples. First, they were asked to taste and rate their overall liking of each sample using a 9-point hedonic scale $(1=$ extremely dislike, $9=$ extremely like). Secondly, they indicated their emotion and wellness responses to each sample using the scales, as described above. Consumers were seated separately and were instructed not to interact with other participants.

Table 3. Selected emotional and wellness terms relevant to herbal drinks.

\begin{tabular}{|c|c|c|}
\hline Term Categories & English & Thai \\
\hline Positive emotional terms & Active & รู้สึกกระฉับกระเฉง \\
\hline \multirow[t]{6}{*}{ (7 terms) } & Energetic & รู้สึกมีพลัง \\
\hline & Good & รู้สึกดี \\
\hline & Happy & รู้สึกมีความสุข \\
\hline & Polite & รู้สึกสสุภาพ \\
\hline & Satisfied & รู้สึกพึงพอใจ \\
\hline & Warm & รู้สึกอบอุ่น \\
\hline Negative emotional terms & Bored & รู้สึกเบื่อ \\
\hline \multirow[t]{2}{*}{ (3 terms) } & Disgusted & รู้รูึสึกน่ารังเกียจ \\
\hline & Worried & รู้สึกกกังวล \\
\hline \multirow{5}{*}{$\begin{array}{l}\text { Wellness terms } \\
\qquad(5 \text { terms })\end{array}$} & Comforted & รู้สึกสบาย \\
\hline & Healthy & มีสุขภาพดี \\
\hline & Invigorated & มีชีวิตชีวา \\
\hline & Relaxed & รู้สูึกผ่อนคลาย \\
\hline & Refreshed & รู้สึกสดชื่น \\
\hline
\end{tabular}

Table 4. Demographic characteristics of participants for the six test designs.

\begin{tabular}{|c|c|c|c|c|c|c|c|}
\hline Test Design & Test 1 & Test 2 & Test 3 & Test 4 & Test 5 & Test 6 & Total \\
\hline Emotion and wellness items & Words & Sentences & Words & Sentences & Words & Sentences & \\
\hline Measurement method & Rating & Rating & CATA & CATA & RATA & RATA & \\
\hline $\begin{array}{c}\text { Number of participants } \\
\text { Gender }(\%)^{1}\end{array}$ & 209 & 208 & 209 & 207 & 208 & 211 & 1252 \\
\hline Male & 49.8 & 49.5 & 49.3 & 49.3 & 49.0 & 48.8 & 49.3 \\
\hline Female & 50.2 & 50.5 & 50.7 & 50.7 & 51.0 & 51.2 & 50.7 \\
\hline \multicolumn{8}{|l|}{ Age (\%) ${ }^{1}$} \\
\hline 18-21 years & 24.9 & 24.5 & 24.4 & 26.6 & 25.2 & 27.5 & 25.6 \\
\hline $22-40$ years & 24.9 & 26.0 & 25.8 & 24.2 & 26.5 & 24.6 & 25.2 \\
\hline $41-59$ years & 25.4 & 25.5 & 25.8 & 25.1 & 24.4 & 24.2 & 25.1 \\
\hline$\geq 60$ years & 24.8 & 24.0 & 24.0 & 24.1 & 23.9 & 23.7 & 24.1 \\
\hline \multicolumn{8}{|l|}{ Region of Thailand (\%) ${ }^{1}$} \\
\hline Northern & 20.1 & 20.2 & 21.1 & 18.8 & 20.7 & 19.4 & 20.1 \\
\hline Central & 22.5 & 20.7 & 19.1 & 24.2 & 25.0 & 26.6 & 23.0 \\
\hline Northeastern & 34.0 & 36.0 & 33.5 & 36.7 & 31.7 & 34.6 & 34.4 \\
\hline Southern & 23.4 & 23.1 & 26.3 & 20.3 & 22.6 & 19.4 & 22.5 \\
\hline
\end{tabular}

${ }^{1}$ Percentage within each test design. 


\subsection{Statistical Analysis}

Analysis of variance (ANOVA) and Duncan's multiple range test (DMRT) were performed on the overall liking scores to determine significant overall and pairwise differences among the five herbal drinks and six test designs.

For rating scores, the mean score of each emotion and wellness term was calculated from consumer responses. For CATA data, a frequency count was calculated by counting the number of consumers who chose the terms for each sample. RATA data were analyzed using one of two approaches [26]: frequency of selection (RATA frequency) or weighted frequency of selection (RATA score). RATA frequency was counted from the number of consumers who chose the terms for each sample. RATA scores were calculated by summing up the scores that the consumers rated for each selected term (0-5 scoring; $0=$ 'not selected', $1=$ 'slightly', and $5=$ 'extremely') [27] and dividing by the number of participating consumers.

Both rating and RATA scores (when treated as a continuous outcome) were analyzed using ANOVA to determine the emotion and wellness terms with significant differences among the five herbal drinks. Next, the significant terms were analyzed using DMRT for multiple comparisons [28]. Principal component analysis (PCA) was performed to identify the emotion and wellness terms that contributed to overall differences among the herbal drinks and to classify the products based on emotion and wellness responses. Overall product differences were determined using multivariate analysis of variance (MANOVA) based on emotion and wellness responses, followed by discriminant analysis (DA) to identify the important terms of group discrimination.

CATA and RATA frequency counts were analyzed following the recommendation of Meyners et al. (2016) [27]. Cochran's $Q$ test was carried out to determine the differences among herbal drink samples for each emotion or wellness response, and the sign test was used for pairwise comparison. Correspondence analysis (CA) based on Chi-square distances was used to identify the emotion and wellness terms that correlated to overall differences among the sampled herbal drinks. DA was performed to identify the important terms of group discrimination.

All statistical analyses were performed at 5\% significance level using the SPSS 19 software (SPSS Inc., Chicago, IL, USA), except DA, which was carried out using XLSTAT ${ }^{\circledR}$ Basic version 2019 software (Addinsoft; New York, NY, USA).

\section{Results and Discussion}

\subsection{Overall Liking of Herbal Drink Samples}

Regarding the overall liking scores of the five herbal drinks from six test designs, the data were analyzed by ANOVA and DMRT (Table 5). In each test, significant differences in overall liking were observed $(p<0.05)$ among the five herbal drinks, indicating that consumers liked the five herbal drinks to varying degrees. In general, roselle and chrysanthemum drinks were similarly well-liked (7.11-7.49) and were found to be more acceptable than the three other herbal drinks (6.48-6.87 for ginger and Jubliang; 6.62-7.06 for Krachai Dam). For overall liking evaluation, different groups of consumers participated in each test design. Homogeneity of hedonic perception should be determined before comparing the different questionnaire designs. When comparing the overall liking score of each herbal drink among different consumers from the six test-design groups, no significant differences were found. This implies that the consumer population in this study was homogenous in terms of hedonic perception of the test samples. Thus, the subsequent emotion and wellness results of the six test designs could be compared since overall liking and emotion terms may be correlated, depending on product, product category, demographics, and psychographics [5].

\subsection{Emotion and Wellness Profiles of Herbal Drinks from Different Test Designs}

As seen from Tables 6 and 7, the type of herbal drink significantly affected most emotion (except "good", "happy", "bored", "worried", and "disgusted" in some cases) 
and all wellness responses of consumers. Generally, negative emotion responses were rated lower than positive emotion and wellness responses. These findings confirm and extend the positive asymmetry of emotions. According to the theory of positive asymmetry, previous research findings reported that consumers experience primarily positive emotions in response to food and food names [29-31]. In the case of beverages, Calve-Porral et al. [32] found positive asymmetry of emotions in beverage consumption, regardless of the type of beverage. Thus, our findings are in agreement with previous studies.

Table 5. The mean overall liking scores of the five herbal drinks in each test design.

\begin{tabular}{|c|c|c|c|c|c|c|c|}
\hline Test Design ${ }^{1}$ & Roselle & Chrysanthemum & Ginger & Jubliang & $\begin{array}{c}\text { Krachai } \\
\text { Dam }\end{array}$ & F-Value ${ }^{3}$ & $p$-Value ${ }^{3}$ \\
\hline 1 & $7.11^{\mathrm{a}} \pm 1.33$ & $7.32^{\mathrm{a}} \pm 1.35$ & $6.48^{c} \pm 2.03$ & $6.81^{b} \pm 1.28$ & $6.65^{b c} \pm 1.82$ & 11.867 & 0.000 \\
\hline 2 & $7.14^{\mathrm{a}} \pm 1.25$ & $7.29^{\mathrm{a}} \pm 1.23$ & $6.55^{b} \pm 1.98$ & $6.76^{b} \pm 1.39$ & $6.62^{b} \pm 1.79$ & 12.870 & 0.000 \\
\hline 3 & $7.13^{\mathrm{a}} \pm 1.38$ & $7.41^{\mathrm{a}} \pm 1.45$ & $6.59^{b} \pm 2.19$ & $6.81^{b} \pm 1.41$ & $6.85^{b} \pm 1.83$ & 9.983 & 0.000 \\
\hline 4 & $7.13^{b} \pm 1.40$ & $7.46^{\mathrm{a}} \pm 1.21$ & $6.48^{c} \pm 2.08$ & $6.48^{c} \pm 1.73$ & $6.74^{\mathrm{c}} \pm 1.81$ & 19.866 & 0.000 \\
\hline 5 & $7.18^{\mathrm{a}} \pm 1.33$ & $7.35^{\mathrm{a}} \pm 1.42$ & $6.81^{b} \pm 2.04$ & $6.87^{b} \pm 1.51$ & $7.06^{\mathrm{ab}} \pm 1.74$ & 4.841 & 0.001 \\
\hline 6 & $7.23^{\mathrm{a}} \pm 1.28$ & $7.49^{\mathrm{a}} \pm 1.22$ & $6.86^{b} \pm 1.96$ & $6.79^{b} \pm 1.59$ & $6.64^{\mathrm{c}} \pm 2.01$ & 13.335 & 0.000 \\
\hline
\end{tabular}

\begin{tabular}{cccccccc}
\hline F-value $^{2}$ & 0.179 & 0.753 & 1.439 & 1.478 & 1.184 & - \\
\hline$p$-value $^{2}$ & 0.970 & 0.584 & 0.208 & 0.195 & 0.315 & - \\
\hline
\end{tabular}

Mean \pm SD from 1252 consumer responses based on a 9-point hedonic scale. ${ }^{\text {a-c }}$ Different letters in the same row indicate significant differences across samples according to Duncan's multiple range test $(p<0.05) .{ }^{1}$ Test 1 rating, words; Test 2 rating, sentences; Test 3 CATA, words; Test 4 CATA, sentences; Test 5 RATA, words; Test 6 RATA, sentences. ${ }^{2}$ F-value and $p$-value in ANOVA were used to assess whether there is a difference among the six test designs. ${ }^{3} \mathrm{~F}$-value and $p$-value in ANOVA were used to assess whether there is a difference among the five herbal drinks.

Table 6. Mean \pm standard deviations of overall liking ${ }^{1}$ and emotion/wellness responses elicited by five herbal drinks in rating ${ }^{2}$ and RATA $^{3}$ questionnaires.

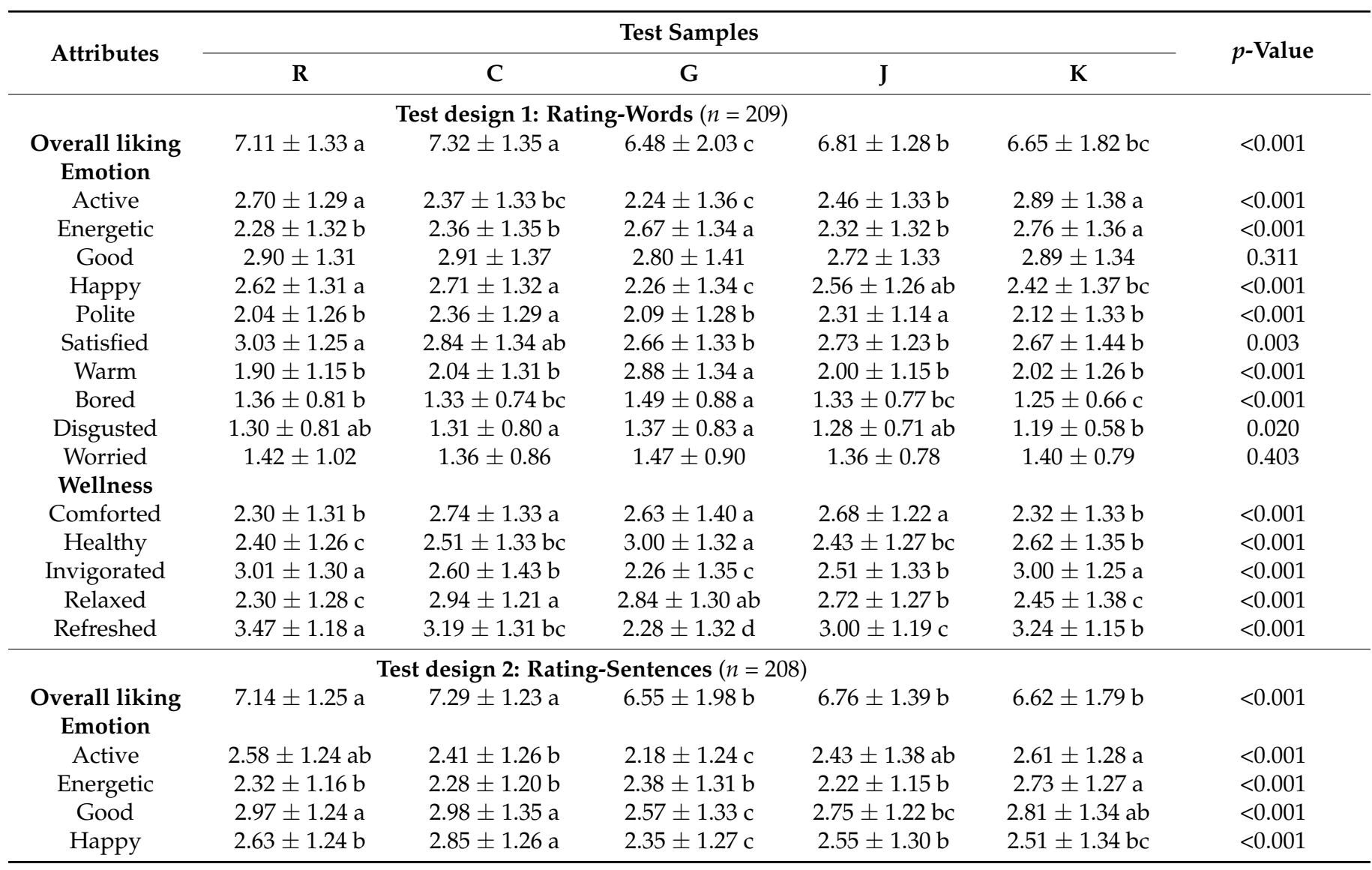


Table 6. Cont.

\begin{tabular}{|c|c|c|c|c|c|c|}
\hline \multirow{2}{*}{ Attributes } & \multicolumn{5}{|c|}{ Test Samples } & \multirow{2}{*}{$p$-Value } \\
\hline & $\mathbf{R}$ & $\mathrm{C}$ & G & $\mathbf{J}$ & $\mathbf{K}$ & \\
\hline Polite & $2.08 \pm 1.17 c$ & $2.49 \pm 1.31 \mathrm{a}$ & $2.03 \pm 1.15 c$ & $2.27 \pm 1.18 \mathrm{~b}$ & $2.00 \pm 1.17 c$ & $<0.001$ \\
\hline Satisfied & $2.94 \pm 1.26 \mathrm{a}$ & $2.89 \pm 1.26 \mathrm{a}$ & $2.49 \pm 1.22 \mathrm{~b}$ & $2.78 \pm 1.26 \mathrm{a}$ & $2.74 \pm 1.38 \mathrm{a}$ & $<0.001$ \\
\hline Warm & $2.11 \pm 1.19 \mathrm{~b}$ & $2.19 \pm 1.23 b$ & $2.81 \pm 1.30 \mathrm{a}$ & $2.08 \pm 1.16 \mathrm{~b}$ & $2.08 \pm 1.20 \mathrm{~b}$ & $<0.001$ \\
\hline Bored & $1.47 \pm 0.94 \mathrm{a}$ & $1.29 \pm 0.68 b$ & $1.34 \pm 0.71 \mathrm{ab}$ & $1.43 \pm 0.83 \mathrm{a}$ & $1.43 \pm 0.87 \mathrm{a}$ & 0.034 \\
\hline Disgusted & $1.31 \pm 0.74 b c$ & $1.24 \pm 0.62 c$ & $1.42 \pm 0.85 \mathrm{a}$ & $1.27 \pm 0.65 c$ & $1.39 \pm 0.85 \mathrm{ab}$ & 0.002 \\
\hline Worried & $1.40 \pm 0.83$ & $1.37 \pm 0.81$ & $1.38 \pm 0.78$ & $1.43 \pm 0.83$ & $1.50 \pm 0.86$ & 0.119 \\
\hline \multicolumn{7}{|l|}{ Wellness } \\
\hline Comforted & $2.54 \pm 1.27 c$ & $3.07 \pm 1.28 \mathrm{a}$ & $2.54 \pm 1.31 c$ & $2.75 \pm 1.17 b$ & $2.43 \pm 1.37 c$ & $<0.001$ \\
\hline Healthy & $2.53 \pm 1.26 \mathrm{~b}$ & $2.56 \pm 1.29 \mathrm{~b}$ & $2.99 \pm 1.26 \mathrm{a}$ & $2.47 \pm 1.30 \mathrm{~b}$ & $2.63 \pm 1.26 \mathrm{~b}$ & $<0.001$ \\
\hline Invigorated & $3.08 \pm 1.15 \mathrm{a}$ & $2.88 \pm 1.32 \mathrm{~b}$ & $2.27 \pm 1.23 c$ & $2.70 \pm 1.25 b$ & $2.87 \pm 1.28 \mathrm{~b}$ & $<0.001$ \\
\hline Relaxed & $2.46 \pm 1.26 \mathrm{~b}$ & $2.79 \pm 1.27 \mathrm{a}$ & $2.56 \pm 1.28 \mathrm{~b}$ & $2.51 \pm 1.29 b$ & $2.25 \pm 1.30 c$ & $<0.001$ \\
\hline Refreshed & $3.32 \pm 1.12 \mathrm{a}$ & $3.52 \pm 1.16 \mathrm{a}$ & $2.45 \pm 1.27 c$ & $3.03 \pm 1.19 b$ & $3.06 \pm 1.19 b$ & $<0.001$ \\
\hline \multicolumn{7}{|c|}{ Test design 5: RATA-Words $(n=208)$} \\
\hline $\begin{array}{l}\text { Overall liking } \\
\text { Emotion }\end{array}$ & $7.18 \pm 1.33 \mathrm{a}$ & $7.35 \pm 1.42 \mathrm{a}$ & $6.81 \pm 2.04 b$ & $6.87 \pm 1.51 b$ & $7.06 \pm 1.74 \mathrm{ab}$ & $<0.001$ \\
\hline Active & $1.83 \pm 1.73 \mathrm{~b}$ & $1.40 \pm 1.81 \mathrm{c}$ & $1.33 \pm 1.73 c$ & $1.67 \pm 1.85 b$ & $2.20 \pm 1.97 \mathrm{a}$ & $<0.001$ \\
\hline Energetic & $1.68 \pm 1.79 \mathrm{ab}$ & $1.53 \pm 1.85 \mathrm{bc}$ & $1.60 \pm 1.82 \mathrm{bc}$ & $1.39 \pm 1.75 c$ & $1.91 \pm 1.89 \mathrm{a}$ & 0.001 \\
\hline Good & $2.35 \pm 1.80 \mathrm{a}$ & $2.15 \pm 1.96 \mathrm{ab}$ & $1.96 \pm 1.86 \mathrm{c}$ & $1.90 \pm 1.83 c$ & $2.05 \pm 1.94 \mathrm{ab}$ & 0.023 \\
\hline Happy & $1.88 \pm 1.77 \mathrm{a}$ & $1.95 \pm 1.92 \mathrm{a}$ & $1.37 \pm 1.71 \mathrm{~b}$ & $1.61 \pm 1.78 \mathrm{~b}$ & $1.50 \pm 1.83 \mathrm{~b}$ & $<0.001$ \\
\hline Polite & $1.42 \pm 1.79 b$ & $1.72 \pm 1.79 \mathrm{a}$ & $1.13 \pm 1.63 c$ & $1.28 \pm 1.64 \mathrm{bc}$ & $1.08 \pm 1.62 \mathrm{c}$ & $<0.001$ \\
\hline Satisfied & $2.26 \pm 1.80 \mathrm{a}$ & $2.06 \pm 1.92 \mathrm{ab}$ & $1.79 \pm 1.76 \mathrm{~b}$ & $2.25 \pm 1.79 \mathrm{a}$ & $2.00 \pm 1.96 \mathrm{ab}$ & 0.008 \\
\hline Warm & $1.15 \pm 1.56 \mathrm{~b}$ & $1.20 \pm 1.66 \mathrm{~b}$ & $2.22 \pm 1.98 \mathrm{a}$ & $0.97 \pm 1.52 \mathrm{~b}$ & $1.08 \pm 1.66 \mathrm{~b}$ & $<0.001$ \\
\hline Bored & $0.47 \pm 1.01 \mathrm{a}$ & $0.41 \pm 1.04 \mathrm{a}$ & $0.44 \pm 0.99 a$ & $0.34 \pm 0.89 \mathrm{ab}$ & $0.24 \pm 0.80 \mathrm{~b}$ & 0.004 \\
\hline Disgusted & $0.26 \pm 0.81 b$ & $0.26 \pm 0.86 b$ & $0.41 \pm 1.01 \mathrm{a}$ & $0.20 \pm 0.75 b$ & $0.21 \pm 0.76 b$ & 0.009 \\
\hline $\begin{array}{l}\text { Worried } \\
\text { Wellness }\end{array}$ & $0.56 \pm 1.22 \mathrm{a}$ & $0.41 \pm 1.05 \mathrm{~b}$ & $0.40 \pm 1.09 \mathrm{~b}$ & $0.30 \pm 0.87 \mathrm{~b}$ & $0.38 \pm 0.95 b$ & 0.014 \\
\hline Comforted & $1.71 \pm 1.80 \mathrm{~b}$ & $2.05 \pm 1.87 \mathrm{a}$ & $1.59 \pm 1.82 \mathrm{bc}$ & $1.72 \pm 1.74 \mathrm{~b}$ & $1.35 \pm 1.79 c$ & $<0.001$ \\
\hline Healthy & $1.73 \pm 1.78 \mathrm{bc}$ & $1.56 \pm 1.86 \mathrm{c}$ & $2.36 \pm 1.94 \mathrm{a}$ & $1.59 \pm 1.78 c$ & $1.90 \pm 1.84 \mathrm{~b}$ & $<0.001$ \\
\hline Invigorated & $2.39 \pm 1.71 \mathrm{a}$ & $1.69 \pm 1.89 \mathrm{~b}$ & $1.38 \pm 1.74 \mathrm{c}$ & $1.84 \pm 1.86 \mathrm{~b}$ & $2.20 \pm 1.90 \mathrm{a}$ & $<0.001$ \\
\hline Relaxed & $2.06 \pm 1.79 \mathrm{ab}$ & $2.22 \pm 1.85 \mathrm{a}$ & $2.03 \pm 1.85 \mathrm{ab}$ & $1.90 \pm 1.82 \mathrm{~b}$ & $1.54 \pm 1.81 \mathrm{c}$ & $<0.001$ \\
\hline Refreshed & $3.20 \pm 1.46 \mathrm{a}$ & $2.96 \pm 1.74 \mathrm{ab}$ & $1.86 \pm 1.90 \mathrm{~d}$ & $2.54 \pm 1.71 \mathrm{c}$ & $2.67 \pm 1.86 b c$ & $<0.001$ \\
\hline \multicolumn{7}{|c|}{ Test design 6: RATA-Sentences $(n=211)$} \\
\hline $\begin{array}{l}\text { Overall liking } \\
\text { Emotion }\end{array}$ & $7.23 \pm 1.28 \mathrm{a}$ & $7.49 \pm 1.22 \mathrm{a}$ & $6.86 \pm 1.96 b$ & $6.79 \pm 1.59 \mathrm{~b}$ & $6.64 \pm 2.01 b$ & $<0.001$ \\
\hline Active & $1.97 \pm 1.76 \mathrm{a}$ & $1.71 \pm 1.79 \mathrm{~b}$ & $1.49 \pm 1.68 \mathrm{~b}$ & $1.54 \pm 1.67 \mathrm{~b}$ & $1.93 \pm 1.76 \mathrm{a}$ & $<0.001$ \\
\hline Energetic & $1.78 \pm 1.69 \mathrm{~b}$ & $1.47 \pm 1.68 c$ & $1.68 \pm 1.63 \mathrm{bc}$ & $1.51 \pm 1.68 \mathrm{c}$ & $2.07 \pm 1.70 \mathrm{a}$ & $<0.001$ \\
\hline Good & $2.56 \pm 1.70 \mathrm{a}$ & $2.43 \pm 1.74 \mathrm{ab}$ & $2.40 \pm 1.71 \mathrm{ab}$ & $2.26 \pm 1.71 \mathrm{c}$ & $2.17 \pm 1.76 \mathrm{c}$ & 0.039 \\
\hline Happy & $2.06 \pm 1.72 \mathrm{a}$ & $2.14 \pm 1.81 \mathrm{a}$ & $1.65 \pm 1.74 \mathrm{~b}$ & $2.08 \pm 1.69 \mathrm{a}$ & $1.41 \pm 1.67 c$ & $<0.001$ \\
\hline Polite & $1.36 \pm 1.59 b$ & $1.62 \pm 1.63 \mathrm{a}$ & $1.29 \pm 1.62 b$ & $1.48 \pm 1.61 \mathrm{ab}$ & $1.31 \pm 1.70 \mathrm{~b}$ & 0.008 \\
\hline Satisfied & $2.57 \pm 1.69 \mathrm{a}$ & $2.42 \pm 1.77 \mathrm{a}$ & $2.28 \pm 1.72 \mathrm{ab}$ & $2.37 \pm 1.65 \mathrm{a}$ & $2.05 \pm 1.84 \mathrm{~b}$ & 0.007 \\
\hline Warm & $1.40 \pm 1.66 \mathrm{~b}$ & $1.31 \pm 1.64 b$ & $2.39 \pm 1.75 \mathrm{a}$ & $1.27 \pm 1.65 b$ & $1.27 \pm 1.62 b$ & $<0.001$ \\
\hline Bored & $0.47 \pm 1.04 \mathrm{ab}$ & $0.34 \pm 0.91 b$ & $0.48 \pm 1.14 \mathrm{ab}$ & $0.38 \pm 0.95 b$ & $0.54 \pm 1.18 \mathrm{a}$ & 0.039 \\
\hline Disgusted & $0.35 \pm 0.96$ & $0.23 \pm 0.79$ & $0.39 \pm 1.10$ & $0.25 \pm 0.79$ & $0.32 \pm 0.93$ & 0.089 \\
\hline $\begin{array}{l}\text { Worried } \\
\text { Wellness }\end{array}$ & $0.34 \pm 0.90 c$ & $0.36 \pm 1.03 c$ & $0.44 \pm 1.16 b c$ & $0.54 \pm 1.15 \mathrm{ab}$ & $0.64 \pm 1.18 \mathrm{a}$ & 0.001 \\
\hline Comforted & $1.94 \pm 1.76 \mathrm{~b}$ & $2.13 \pm 1.79 \mathrm{ab}$ & $1.98 \pm 1.74 b$ & $2.27 \pm 1.66 \mathrm{a}$ & $1.61 \pm 1.72 \mathrm{c}$ & $<0.001$ \\
\hline Healthy & $1.86 \pm 1.76 c$ & $1.74 \pm 1.78 \mathrm{c}$ & $2.64 \pm 1.71 \mathrm{a}$ & $1.72 \pm 1.78 c$ & $2.19 \pm 1.80 \mathrm{~b}$ & $<0.001$ \\
\hline Invigorated & $2.52 \pm 1.67 \mathrm{a}$ & $1.88 \pm 1.78 \mathrm{~b}$ & $1.58 \pm 1.71 \mathrm{c}$ & $2.02 \pm 1.73 \mathrm{~b}$ & $2.36 \pm 1.74 \mathrm{a}$ & $<0.001$ \\
\hline Relaxed & $1.80 \pm 1.73 \mathrm{bc}$ & $2.14 \pm 1.73 \mathrm{a}$ & $2.00 \pm 1.61 \mathrm{ab}$ & $1.75 \pm 1.65 c$ & $1.50 \pm 1.71 \mathrm{~d}$ & $<0.001$ \\
\hline Refreshed & $3.09 \pm 1.44 \mathrm{a}$ & $3.07 \pm 1.52 \mathrm{a}$ & $1.93 \pm 1.81 \mathrm{c}$ & $2.79 \pm 1.36 b$ & $2.90 \pm 1.55 \mathrm{ab}$ & $<0.001$ \\
\hline
\end{tabular}

${ }^{1}$ Mean \pm SD of overall liking scores based on a 9-points hedonic scale $(1=$ extremely dislike to $5=$ extremely like). ${ }^{2}$ Mean \pm SD of rating scores based on a 5 -points scale $\left(1=\right.$ not at all to $5=$ extremely). ${ }^{3}$ Mean \pm SD of RATA rating scores based on a 5 -points scale $(1=$ slightly to $5=$ extremely). a-d Mean values in the same row followed by different letters are significantly different $(p<0.05)$. Note: $\mathrm{R}=$ Roselle drink; $\mathrm{C}=\mathrm{Chrysanthemum}$ drink; $\mathrm{G}=$ Ginger drink; J = Jubliang drink; $\mathrm{K}$ = Krachai Dam drink. 
Table 7. Mean \pm standard deviations of overall liking ${ }^{1}$ and frequency counts of emotion/wellness responses elicited by five herbal drinks in CATA ${ }^{2}$ and RATA ${ }^{2}$ questionnaires.

\begin{tabular}{|c|c|c|c|c|c|c|c|}
\hline \multirow{2}{*}{ Attributes } & \multicolumn{5}{|c|}{ Test Samples } & \multirow{2}{*}{ Cochran's Q } & \multirow{2}{*}{$p$-Value } \\
\hline & $\mathbf{R}$ & $\mathrm{C}$ & G & $\mathbf{J}$ & $\mathbf{K}$ & & \\
\hline \multicolumn{8}{|c|}{ Test design 3: CATA-Words $(n=209)$} \\
\hline $\begin{array}{l}\text { Overall liking } \\
\text { Emotion }\end{array}$ & $7.13 \pm 1.38 \mathrm{a}$ & $7.41 \pm 1.45 \mathrm{a}$ & $6.59 \pm 2.19 b$ & $6.81 \pm 1.41 b$ & $6.85 \pm 1.83 \mathrm{~b}$ & & $<0.001$ \\
\hline Active & 58 & 41 & 42 & 48 & 95 & 68.962 & $<0.001$ \\
\hline Energetic & 39 & 41 & 74 & 45 & 68 & 45.305 & $<0.001$ \\
\hline Good & 79 & 91 & 74 & 81 & 84 & 4.603 & 0.331 \\
\hline Happy & 46 & 56 & 40 & 45 & 52 & 8.082 & 0.089 \\
\hline Polite & 28 & 48 & 36 & 31 & 27 & 16.994 & 0.002 \\
\hline Satisfied & 69 & 79 & 52 & 66 & 63 & 13.338 & 0.010 \\
\hline Warm & 18 & 24 & 100 & 27 & 26 & 222.749 & $<0.001$ \\
\hline Bored & 14 & 21 & 23 & 18 & 13 & 14.667 & 0.005 \\
\hline Disgusted & 14 & 13 & 24 & 13 & 16 & 21.500 & $<0.001$ \\
\hline Worried & 24 & 19 & 23 & 21 & 26 & 3.476 & 0.482 \\
\hline \multicolumn{8}{|l|}{ Wellness } \\
\hline Comforted & 45 & 81 & 62 & 79 & 43 & 42.904 & $<0.001$ \\
\hline Healthy & 43 & 54 & 106 & 65 & 67 & 71.609 & $<0.001$ \\
\hline Invigorated & 84 & 62 & 39 & 70 & 93 & 52.529 & $<0.001$ \\
\hline Relaxed & 60 & 104 & 77 & 86 & 60 & 40.209 & $<0.001$ \\
\hline Refreshed & 156 & 143 & 66 & 137 & 114 & 119.685 & $<0.001$ \\
\hline \multicolumn{8}{|c|}{ Test design 4: CATA-Sentences $(n=207)$} \\
\hline $\begin{array}{l}\text { Overall liking } \\
\text { Emotion }\end{array}$ & $7.13 \pm 1.40 \mathrm{~b}$ & $7.46 \pm 1.21 \mathrm{a}$ & $6.48 \pm 2.08 c$ & $6.48 \pm 1.73 c$ & $6.74 \pm 1.81 c$ & & $<0.001$ \\
\hline Active & 53 & 47 & 40 & 42 & 90 & 68.784 & $<0.001$ \\
\hline Energetic & 44 & 33 & 64 & 30 & 72 & 65.502 & $<0.001$ \\
\hline Good & 85 & 108 & 79 & 89 & 81 & 17.227 & 0.002 \\
\hline Happy & 64 & 82 & 46 & 53 & 53 & 36.553 & $<0.001$ \\
\hline Polite & 20 & 47 & 32 & 41 & 16 & 45.342 & $<0.001$ \\
\hline Satisfied & 72 & 73 & 49 & 70 & 67 & 14.916 & 0.005 \\
\hline Warm & 22 & 35 & 82 & 41 & 33 & 106.995 & $<0.001$ \\
\hline Bored & 18 & 19 & 22 & 24 & 21 & 4.750 & 0.314 \\
\hline Disgusted & 17 & 16 & 25 & 23 & 22 & 14.571 & 0.006 \\
\hline Worried & 15 & 18 & 20 & 21 & 22 & 5.500 & 0.240 \\
\hline \multicolumn{8}{|l|}{ Wellness } \\
\hline Comforted & 75 & 102 & 76 & 95 & 66 & 27.069 & $<0.001$ \\
\hline Healthy & 61 & 115 & 69 & 63 & 68 & 65.954 & $<0.001$ \\
\hline Invigorated & 81 & 77 & 48 & 79 & 105 & 46.724 & $<0.001$ \\
\hline Relaxed & 71 & 77 & 77 & 58 & 47 & 24.281 & $<0.001$ \\
\hline Refreshed & 160 & 141 & 77 & 123 & 124 & 89.387 & $<0.001$ \\
\hline \multicolumn{8}{|c|}{ Test design 5: RATA-Words $(n=208)$} \\
\hline $\begin{array}{l}\text { Overall liking } \\
\text { Emotion }\end{array}$ & $7.18 \pm 1.33 \mathrm{a}$ & $7.35 \pm 1.42 \mathrm{a}$ & $6.81 \pm 2.04 b$ & $6.87 \pm 1.51 \mathrm{~b}$ & $7.06 \pm 1.74 \mathrm{ab}$ & & $<0.001$ \\
\hline Active & 116 & 83 & 83 & 102 & 124 & 52.089 & $<0.001$ \\
\hline Energetic & 104 & 90 & 97 & 86 & 115 & 21.243 & $<0.001$ \\
\hline Good & 137 & 120 & 118 & 114 & 118 & 10.919 & 0.027 \\
\hline Happy & 116 & 111 & 87 & 100 & 89 & 25.585 & $<0.001$ \\
\hline Polite & 86 & 106 & 71 & 87 & 69 & 39.768 & $<0.001$ \\
\hline Satisfied & 133 & 116 & 113 & 135 & 113 & 15.948 & 0.003 \\
\hline Warm & 78 & 78 & 125 & 64 & 66 & 94.646 & $<0.001$ \\
\hline Bored & 39 & 31 & 36 & 28 & 18 & 21.387 & $<0.001$ \\
\hline Disgusted & 22 & 20 & 32 & 15 & 16 & 17.864 & 0.001 \\
\hline Worried & 40 & 30 & 27 & 24 & 32 & 10.590 & 0.032 \\
\hline Wellness & & & & & & & \\
\hline Comforted & 104 & 120 & 96 & 109 & 82 & 30.556 & $<0.001$ \\
\hline Healthy & 106 & 91 & 131 & 100 & 115 & 34.691 & $<0.001$ \\
\hline
\end{tabular}


Table 7. Cont.

\begin{tabular}{|c|c|c|c|c|c|c|c|}
\hline \multirow{2}{*}{ Attributes } & \multicolumn{5}{|c|}{ Test Samples } & \multirow{2}{*}{ Cochran's Q } & \multirow{2}{*}{$p$-Value } \\
\hline & $\mathbf{R}$ & $\mathrm{C}$ & G & $\mathbf{J}$ & $\mathbf{K}$ & & \\
\hline Invigorated & 145 & 98 & 86 & 110 & 127 & 69.516 & $<0.001$ \\
\hline Relaxed & 125 & 128 & 122 & 116 & 95 & 21.713 & $<0.001$ \\
\hline \multirow[t]{2}{*}{ Refreshed } & 179 & 161 & 109 & 152 & 147 & 81.199 & $<0.001$ \\
\hline & \multicolumn{4}{|c|}{ Test design 6: RATA-Sentences $(n=211)$} & & & \\
\hline $\begin{array}{l}\text { Overall liking } \\
\text { Emotion }\end{array}$ & $7.23 \pm 1.28 \mathrm{a}$ & $7.49 \pm 1.22 \mathrm{a}$ & $6.86 \pm 1.96 b$ & $6.79 \pm 1.59 b$ & $6.64 \pm 2.01 b$ & & $<0.001$ \\
\hline Active & 127 & 109 & 98 & 103 & 126 & 34.400 & $<0.001$ \\
\hline Energetic & 118 & 99 & 116 & 101 & 137 & 41.902 & $<0.001$ \\
\hline Good & 157 & 149 & 151 & 144 & 139 & 6.643 & 0.156 \\
\hline Happy & 133 & 130 & 108 & 135 & 95 & 56.124 & $<0.001$ \\
\hline Polite & 96 & 112 & 88 & 105 & 88 & 22.218 & $<0.001$ \\
\hline Satisfied & 158 & 147 & 144 & 151 & 125 & 20.266 & $<0.001$ \\
\hline Warm & 95 & 90 & 147 & 84 & 89 & 121.892 & $<0.001$ \\
\hline Bored & 38 & 27 & 35 & 31 & 39 & 7.353 & 0.118 \\
\hline Disgusted & 26 & 18 & 26 & 20 & 24 & 4.935 & 0.294 \\
\hline Worried & 28 & 26 & 31 & 42 & 53 & 28.398 & $<0.001$ \\
\hline Wellness & & & & & & & \\
\hline Comforted & 123 & 132 & 127 & 148 & 108 & 36.416 & $<0.001$ \\
\hline Healthy & 119 & 113 & 158 & 107 & 137 & 64.692 & $<0.001$ \\
\hline Invigorated & 158 & 120 & 104 & 129 & 147 & 65.381 & $<0.001$ \\
\hline Relaxed & 116 & 137 & 136 & 118 & 99 & 39.635 & $<0.001$ \\
\hline Refreshed & 184 & 180 & 122 & 181 & 175 & 96.239 & $<0.001$ \\
\hline
\end{tabular}

${ }^{1}$ Mean \pm SD of overall liking scores based on a 9-point hedonic scale $(1=$ extremely dislike, 5 = extremely like). ${ }^{2}$ Frequency counts of selected emotion/wellness terms were analyzed using Cochran's Q test. a-c Mean values in the same row followed by different letters are significantly different $(p<0.05)$. Note: $\mathrm{R}=$ roselle drink; $\mathrm{C}=$ chrysanthemum drink; $\mathrm{G}=$ ginger drink; $\mathrm{J}=$ Jubliang drink; $\mathrm{K}=$ Krachai Dam drink.

When comparing the two formats of questionnaire items-words vs. full sentencessome minor differences, in terms of the number of significant emotion and wellness terms, were observed, depending on the measurement scale used. The number of significant terms for test 1 (rating-words) vs. test 2 (rating-sentences) was 13 vs. 14 for test 5 (RATA/scoreswords) vs. test 6 (RATA/scores-sentences) was 15 vs. 14, for test 3 (CATA-words) vs. test 4 (CATA-sentences) was 12 vs. 13, and for test 5 (RATA/frequency-words) vs. test 6 (RATA/frequency-sentences) was 15 vs. 12. A greater difference was observed between words and sentences in the RATA frequency test format. However, overall, sentence format may not reduce ambiguity over the word-only format in the questionnaire. For terms unfamiliar to consumers (such as "polite"), including a context may help to increase understanding of the specified emotion or wellness state. However, the emotion and wellness terms used in the questionnaire in this study were pre-screened by consumers who regularly consume the herbal drink products. Consumers selected the terms that they were familiar with; therefore, a full sentence in the questionnaire may not provide much more clarity under the conditions of the current study.

The results from three types of measurement methods, including a rating scale, CATA, and RATA, were compared. The RATA data consisted of two types: RATA frequency and RATA score. Hence, the RATA scores were compared with rating scores, and RATA frequencies were compared with CATA frequencies.

To compare rating scores (test 1 ) and RATA scores (test 5) from the word-format questionnaire, mean scores of emotion and wellness responses were plotted on a spider web, as shown in Figure 1. The overall profiles were visually similar, but the ratings from the rating scores were higher than those from the RATA scores. The rating-words profile (test 1 ) in Figure 1A reveals that significant product differences were observed for 13 terms $(p<0.05)$, except for "worried" (1.36-1.47 scores) and "good" (2.72-2.91 scores) terms (Table 6). Interestingly, the "refreshed" term showed higher scores of four herbal drinks 
(roselle, Jubliang, chrysanthemum, and Krachai Dam drink) (3.47, 3.00, 3.19, and 3.24, respectively) than other emotion and wellness terms. Meanwhile, the score of the "warm" term (2.88) was higher for ginger drink than other terms, with a maximum mean-difference value of 0.98 . King et al. [29] suggested that a mean emotional difference of $\geq 0.2$ units (on a 5-point scale) may be of practical value. For the rata-words profile (test 5) in Figure 1B, all 15 terms had significant differences among the five products. Therefore, using the RATA scale with words (Test 5) showed better discrimination ability than the rating scale with words (15 vs. 13) in terms of improving the number of significantly different terms.

(A) Rating-Words

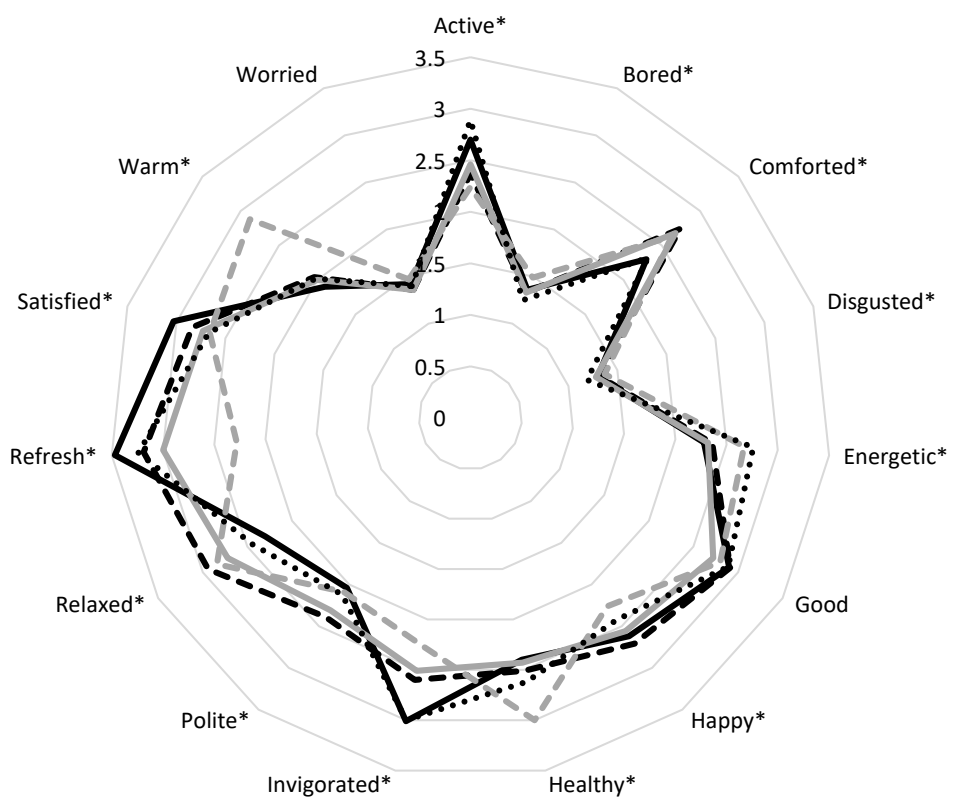

(B) RATA-Words

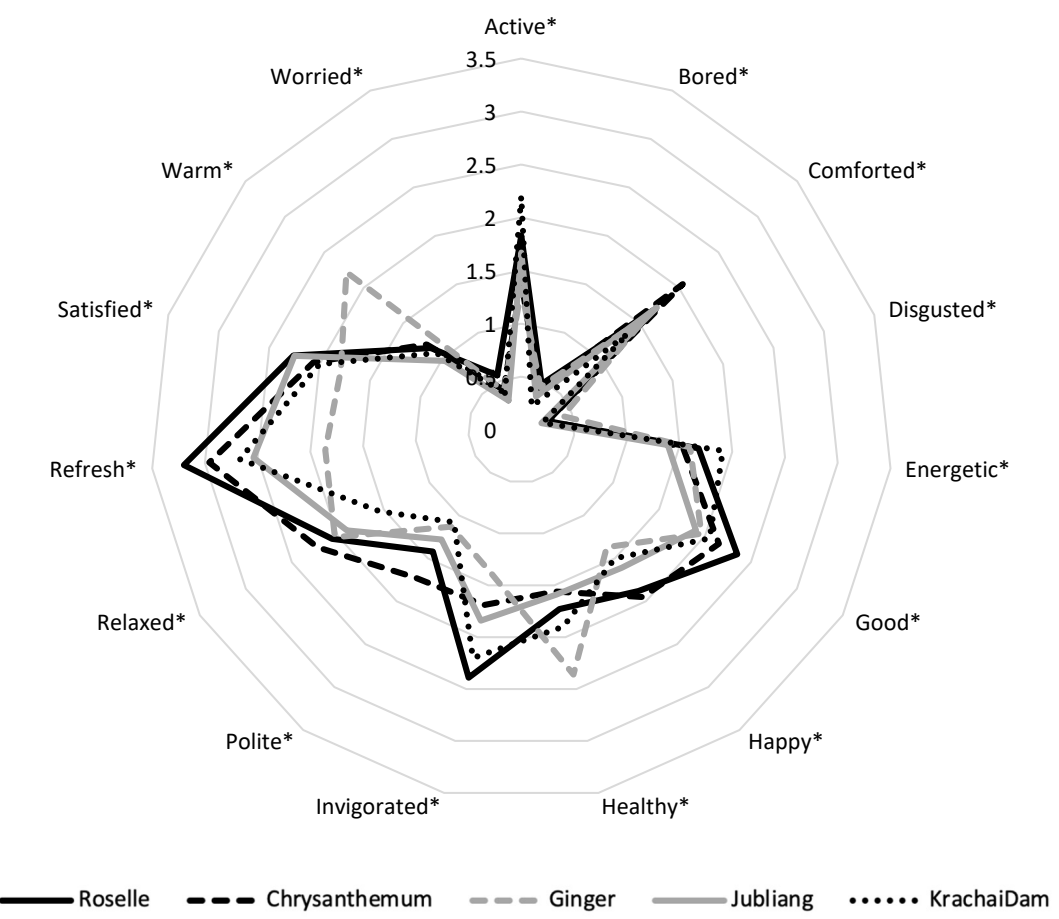

Figure 1. Emotion and wellness profiles of five herbal drinks from the (A) Rating-Words (score 1-5; $n=209$ ) and (B) RATA-Words (score 0-5; $n=208$ ) questionnaires. * indicates significant differences among the five herbal drinks $(p<0.05)$. 
To compare the CATA frequency (test 3 ) and RATA frequency (test 5) from the wordformat questionnaire, emotion and wellness responses, in terms of percentage of the frequency count, were plotted on a line chart, as shown in Figure 2. The two profiles were visually similar, but the frequency counts of the RATA-Words format was higher than those of the CATA-Words format. For CATA-Words in Figure 2A, the "refreshed" term was popular among consumers for roselle $(74.64 \%)$, Jubliang $(65.55 \%)$, chrysanthemum (68.42\%), and Krachai Dam drink (54.55\%), while "good", "happy", and "worried" terms were not significantly different among the five herbal drinks $(p>0.05$; Table 7). For RATAWords in Figure 2B, all 15 terms were significantly different among the five products. Thus, the RATA-Words show a slight improvement in the number of significantly different terms. Therefore, using the RATA scale with words (Test 5) showed slightly better discrimination ability than the CATA scale with words (15 vs. 12) in terms of increasing the number of significant terms.

(A) CATA-Words

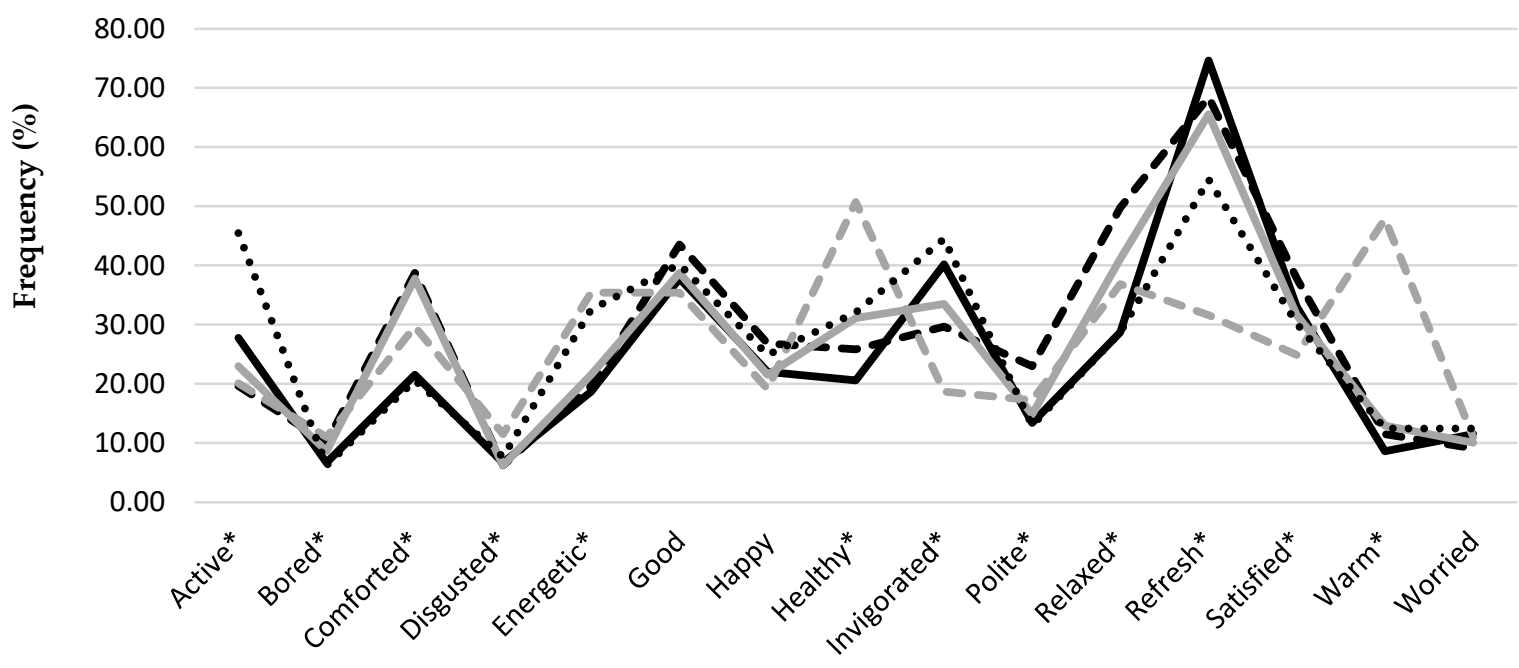

(B) RATA-Words

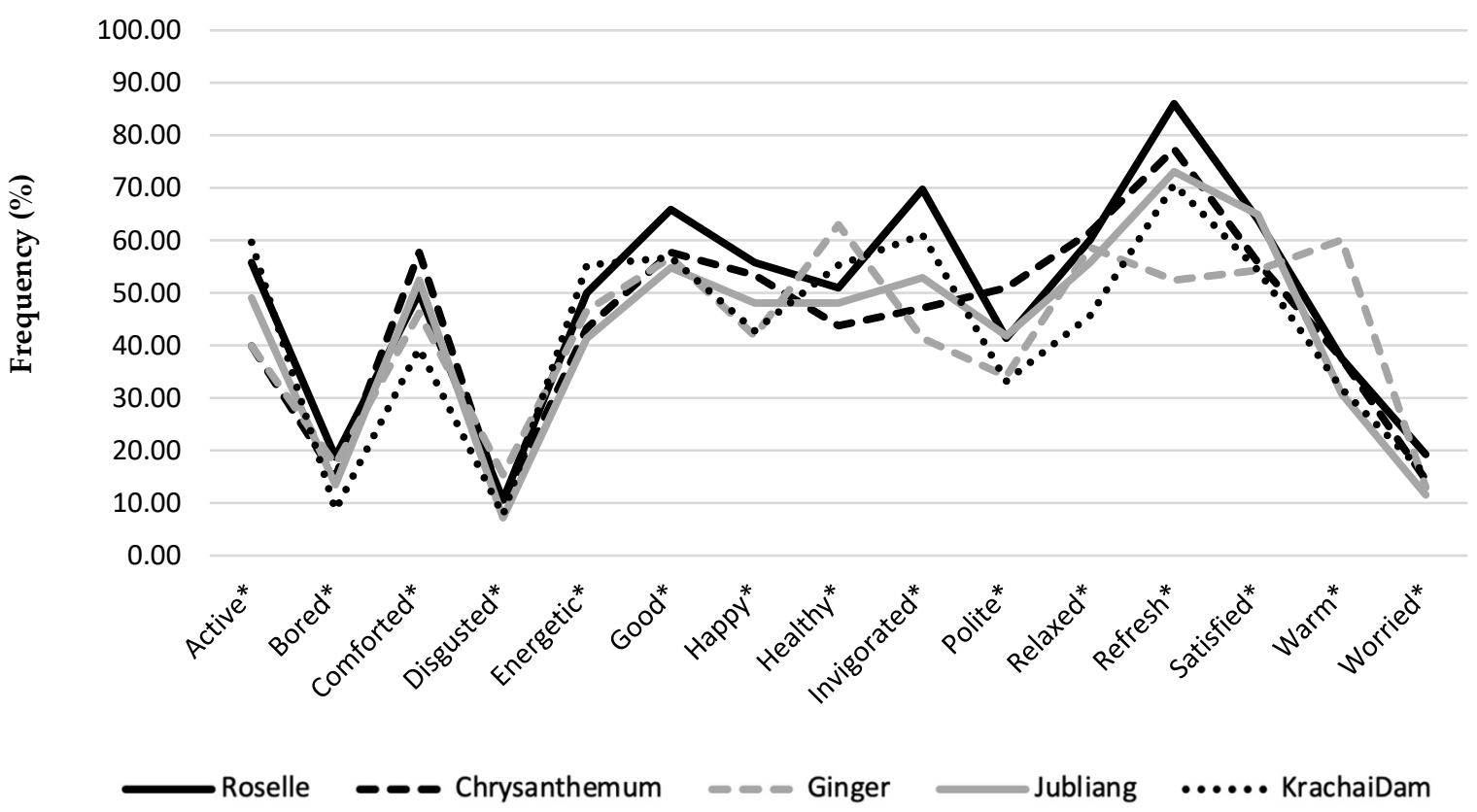

Figure 2. Emotion and wellness profiles of the five herbal drinks from the (A) CATA-Words (test 3; $n=209$ ) and (B) RATA-Words (test 5; $n=208$ ). ${ }^{*}$ indicates significant differences among five herbal drinks $(p<0.05)$. 
Soares et al. [33] mentioned that the word-association (WA) method is used in consumer and marketing research to provide insights on perceptions and attitudes to achieve a deeper understanding of what consumers really think and feel about a product. Many researchers have studied the benefit of herbs, such as roselle, ginger and chrysanthemum, and found that they are a good source of vitamins and minerals and rich in phenolic compounds known to have free-radical-scavenging abilities [34-38]. In this study, consumers selected the "active" and "energetic" emotion terms, as well as the "invigorated", "refreshed", and "relaxed" wellness terms for these five herbal drink products. It is possible that some participants realized the important benefits of these herbal drink; thus, they chose those emotion and wellness terms based on their prior knowledge and consumption experiences. It is known that characteristics of an individual participant may affect the emotion profile of foods [2]; these characteristics include personal preference, past experience, frequency of consumption [30], and culture [39].

\subsection{Discriminating Herbal Drinks Based on Emotion and Wellness Responses}

To determine the overall product differences and which emotion and wellness attributes were mainly responsible for product discrimination, MANOVA and DA were performed (Table 8). When considering the MANOVA results of rating scores and RATA scores, significant differences were observed among the five herbal drinks in terms of emotion and wellness responses $(p<0.0001)$. Based on the first canonical dimension (Can 1), DA identified the "warm" emotion and the "refreshed" wellness terms as the two most discriminating terms among the herbal drinks based on questionnaire responses. However, one additional wellness term ("healthy") was identified in Can 1 for the CATA frequency questionnaire. When considering Can 2, "active" and "comforted" terms significantly contributed to overall product differences. However, RATA scores identified one more wellness term ("relaxed"), while rating scores and CATA frequencies identified two more wellness terms ("relaxed" and "invigorated"). In summary, DA of CATA data revealed the greatest number of terms that discriminated the products, including two positive emotion terms ("warm" and "active") and all five wellness terms ("refreshed", "healthy", "comforted", "relaxed", and "invigorated") with greatest explained variance (93.6\%). This finding (Table 8) is not in agreement with that previously reported based on the results from Tables 6 and 7. One possible explanation is that the former data analysis took into account intercorrelations among emotion and wellness attributes, while the latter did not.

PCA was performed to investigate the relationship between the five herbal drinks and emotion and wellness responses. PCA biplots of rating scores and RATA scores were compared (Figure 3A,B) with the first two PCs (PC1 and PC2), explaining 78.98\% and $77.50 \%$ of total variance, respectively. For rating-words data, the PCA biplots identified four groups of herbal drinks: I-ginger drink, II-Krachai Dam drink, III-roselle drink, and IVchrysanthemum-Jubliang drink (Figure 3A). Ginger drink and roselle drink corresponded with PC1 but in the opposite quadrant, while Krachai Dam drink and chrysanthemumJubliang drink corresponded with PC2 but in the opposite quadrant as well. Ginger drink correlated with "warm", "healthy", "disgusted", and "bored". Roselle drink correlated with "refreshed", "invigorated", and "active". Krachai Dam drink correlated with "energetic" and "worried". Chrysanthemum and Jubliang drinks correlated with "polite" and "comforted".

For RATA-words data, the PCA biplots revealed three groups of herbal drinks: Iginger drink, II-Krachai Dam drink, and III-roselle, chrysanthemum, and Jubliang drinks (Figure 3B). Ginger drink and roselle drink corresponded with PC1, and Krachai Dam drink and Chrysanthemum drink corresponded with PC2, in opposite directions. Jubliang drink corresponded with PC3 (not shown in Figure 3), as it could not be explained using PC1 and PC2. Ginger drink correlated with "warm", "healthy", and "disgusted". Roselle drink correlated with "refreshed", "invigorated", "happy", "satisfied", and "polite". Krachai Dam drink correlated with "energetic" and "active". Chrysanthemum drink correlated with "polite", "comforted", and "bored". Obviously, the "warm" emotion term and the 
"refreshed" wellness term were the most important terms differentiating the hot ginger drink from the cold herbal drinks (Table 8 and Figure 3).

Table 8. Canonical structure $\left(\mathrm{r}^{\prime} \mathrm{s}\right)^{1}$ describing group differences among the five herbal drinks based on emotion and wellness responses obtained from the rating, CATA, and RATA questionnaires.

\begin{tabular}{|c|c|c|c|c|c|c|c|c|}
\hline \multirow{2}{*}{ Responses } & \multicolumn{2}{|c|}{ Rating Scores ${ }^{2}$} & \multicolumn{2}{|c|}{ RATA Scores $^{2}$} & \multicolumn{2}{|c|}{$\begin{array}{c}\text { CATA } \\
\text { Frequencies }^{2}\end{array}$} & \multicolumn{2}{|c|}{$\begin{array}{c}\text { RATA } \\
\text { Frequencies }^{2}\end{array}$} \\
\hline & Can 1 & Can 2 & Can 1 & Can 2 & Can 1 & Can 2 & Can 1 & Can 2 \\
\hline \multicolumn{9}{|l|}{ Emotion } \\
\hline Active & 0.181 & -0.319 & 0.148 & -0.402 & 0.109 & -0.531 & 0.177 & -0.349 \\
\hline Energetic & -0.142 & -0.232 & -0.001 & -0.191 & -0.210 & -0.234 & 0.003 & -0.224 \\
\hline Good & 0.037 & -0.039 & 0.079 & 0.025 & 0.059 & 0.044 & 0.055 & -0.011 \\
\hline Happy & 0.162 & 0.159 & 0.174 & 0.173 & 0.069 & 0.014 & 0.159 & 0.147 \\
\hline Polite & 0.032 & 0.228 & 0.134 & 0.287 & -0.017 & 0.210 & 0.139 & 0.284 \\
\hline Satisfied & 0.117 & 0.028 & 0.148 & 0.008 & 0.120 & 0.111 & 0.114 & 0.035 \\
\hline Warm & -0.457 & -0.020 & -0.465 & 0.137 & -0.648 & 0.036 & -0.461 & 0.114 \\
\hline Bored & -0.122 & 0.041 & -0.027 & 0.161 & -0.071 & 0.127 & -0.043 & 0.140 \\
\hline Disgusted & -0.072 & 0.092 & -0.139 & 0.071 & -0.115 & -0.044 & -0.165 & 0.053 \\
\hline Worried & -0.049 & -0.060 & 0.030 & 0.010 & -0.005 & -0.097 & 0.061 & -0.053 \\
\hline \multicolumn{9}{|l|}{ Wellness } \\
\hline Comforted & -0.091 & 0.316 & 0.075 & 0.316 & -0.013 & 0.409 & 0.080 & 0.302 \\
\hline Healthy & -0.259 & -0.088 & -0.267 & -0.112 & -0.348 & -0.045 & -0.222 & -0.177 \\
\hline Invigorated & 0.282 & -0.315 & 0.264 & -0.281 & 0.248 & -0.303 & 0.293 & -0.284 \\
\hline Relaxed & -0.166 & 0.355 & -0.003 & 0.313 & -0.004 & 0.399 & -0.016 & 0.259 \\
\hline Refreshed & 0.525 & -0.110 & 0.441 & 0.051 & 0.493 & 0.192 & 0.485 & 0.059 \\
\hline$\%$ Cumulative & & & & & & & & \\
\hline $\begin{array}{c}\text { variance } \\
\text { explained }\end{array}$ & 62.5 & 89.0 & 61.7 & 90.7 & 68.5 & 93.6 & 54.4 & 87.5 \\
\hline $\begin{array}{c}\text { MANOVA; } \\
\text { Wilks' Lambda } \\
p \text {-value }\end{array}$ & \multicolumn{2}{|c|}{$<0.0001$} & \multicolumn{2}{|c|}{$<0.0001$} & & & & \\
\hline
\end{tabular}

\footnotetext{
${ }^{1}$ Based on the pooled within-group variance with $p<0.0001$ for rating score, RATA score, CATA frequency, and RATA frequency. Can1 and Can2 refer to the first and second canonical discrimination function, respectively. ${ }^{2}$ Rating scores from Rating-Words question, RATA scores; RATA frequencies from RATA-Words question; CATA frequencies from CATA-Words question.
}

The CA symmetric plots of CATA frequency and RATA frequency were compared (Figure 4A,B). The first two dimensions (Dim1 and Dim2) accounted for $94.79 \%$ and $89.60 \%$ of variance, respectively. CA configuration revealed four groups of herbal drinks: I-ginger drink, II-Krachai Dam drink, III-roselle drink, and IV-chrysanthemum-Jubliang drink. The ginger drink and the other four herbal drinks were clearly separated, on the opposite sides of Dim1 in both CA configurations. However, the CA configurations of CATA data vs. RATA data revealed some slightly different relationships between herbal drinks and emotion and wellness profiles. For CATA data (Figure 4A), ginger drink correlated with "warm", "healthy", and "disgusted". Roselle drink correlated with "refreshed" and "invigorated". Krachai Dam drink correlated with "active". Chrysanthemum and Jubliang drinks correlated with "polite", "relaxed", and "comforted". For RATA data (Figure 4B), ginger drink correlated with "warm" and "disgusted". Roselle drink correlated with "refreshed" and "invigorated". Krachai Dam drink correlated with "energetic" and "active". Chrysanthemum and Jubliang drinks correlated with "polite", "comforted", "relaxed", and "happy".

King et al. [31] evaluated the impact of rating scale and CATA on measurement of emotional responses. They found that the rating scale provided differentiation for more attributes at lower levels of emotional response, while CATA provided greater differentiation at higher levels of emotional frequency for a few select emotions. The rating scale was more sensitive than CATA, but both were found to be acceptable approaches, depending on the objective of the test [31]. In addition, $\mathrm{Ng}$ et al. [6] evaluated the effectiveness of the CATA 
approach compared to intensity scaling for the EsSense ${ }^{\circledR}$ Profile. They reported that the qualitative nature of the data obtained from CATA limited the extent of statistical analysis, making it difficult to draw clear inferential conclusions from the data obtained with the EsSense Profile ${ }^{\circledR}$. Therefore, using a combination of both approaches, RATA was proposed.

(A) Rating-Words

Biplot (axes PC1 and PC2: $78.98 \%$ )

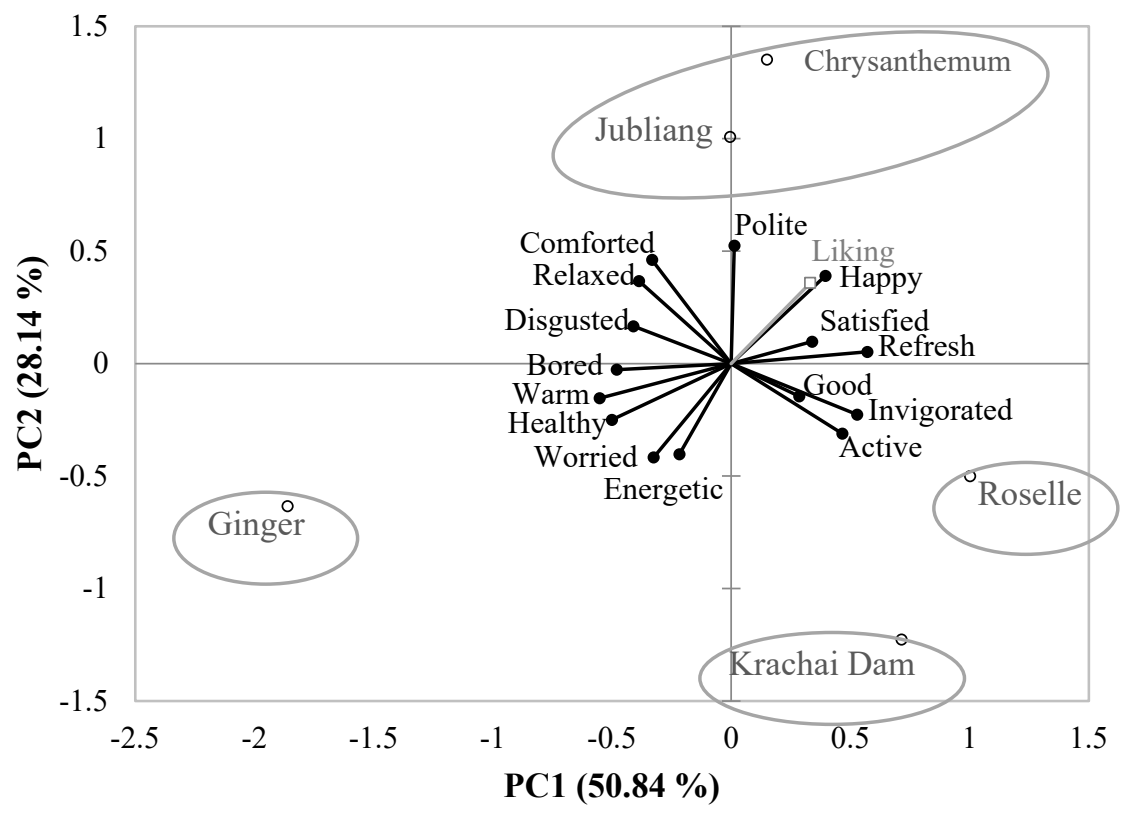

(B) RATA-Words

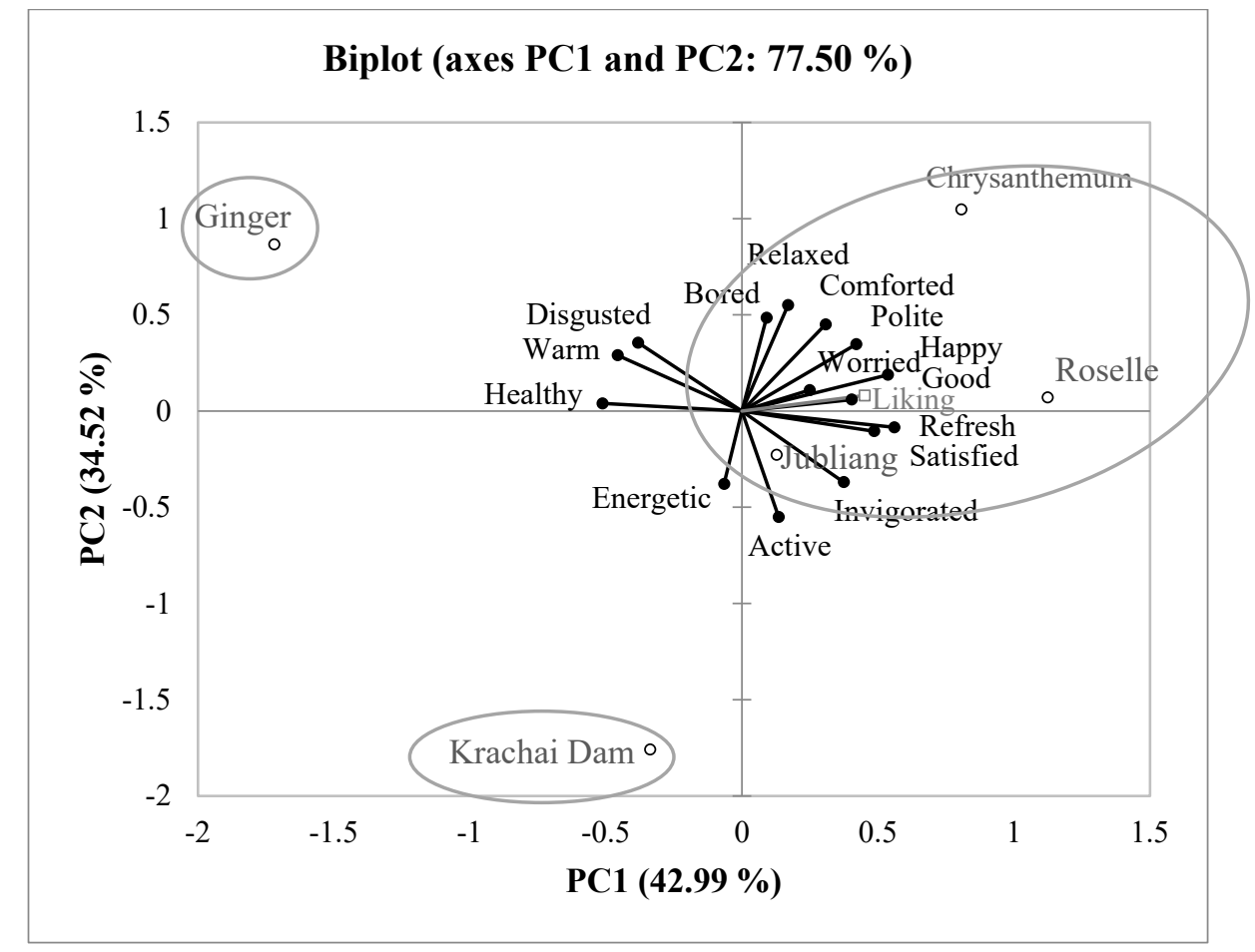

Figure 3. PCA biplots of emotion and wellness responses of the five herbal drinks in the (A) RatingWords and (B) RATA-Words questionnaires. Symbols $(\bigcirc)$ and $(\bullet)$ indicate the position of herbal drinks and emotion-wellness terms, respectively. 


\section{(A) CATA-Words}

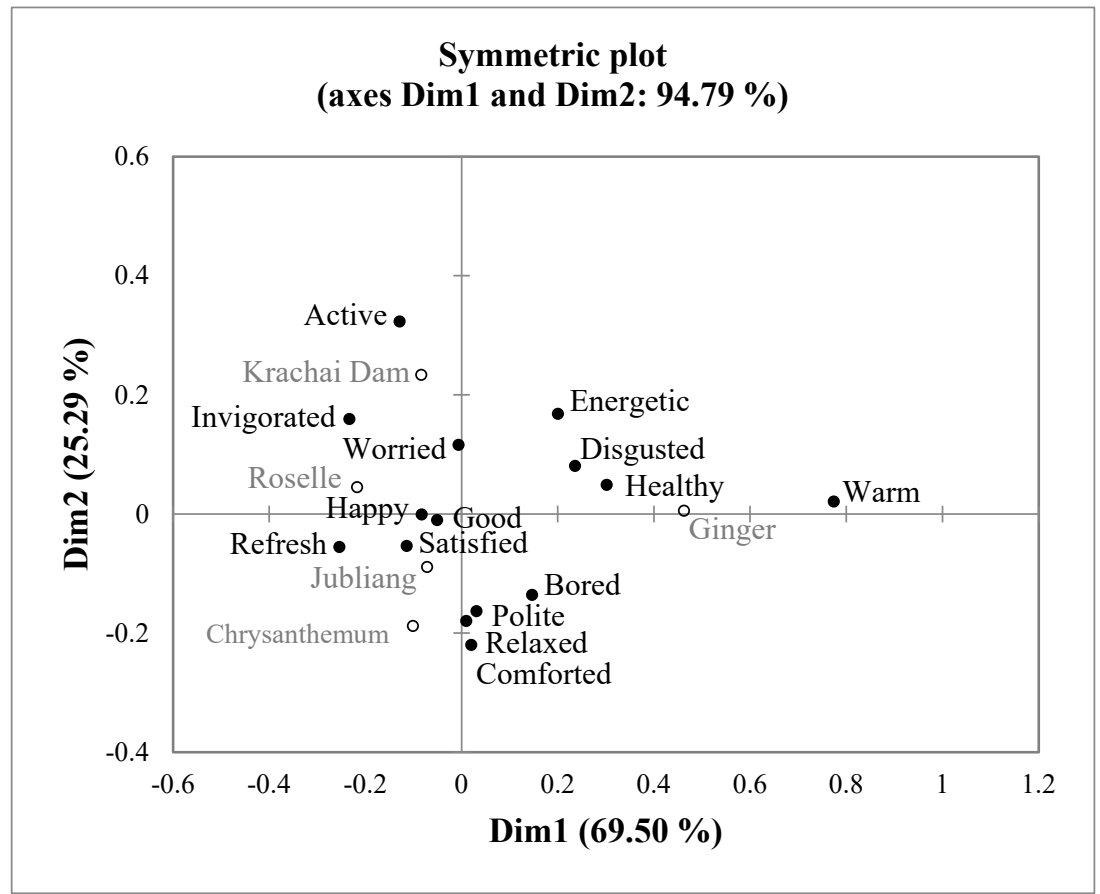

(B) RATA-Words

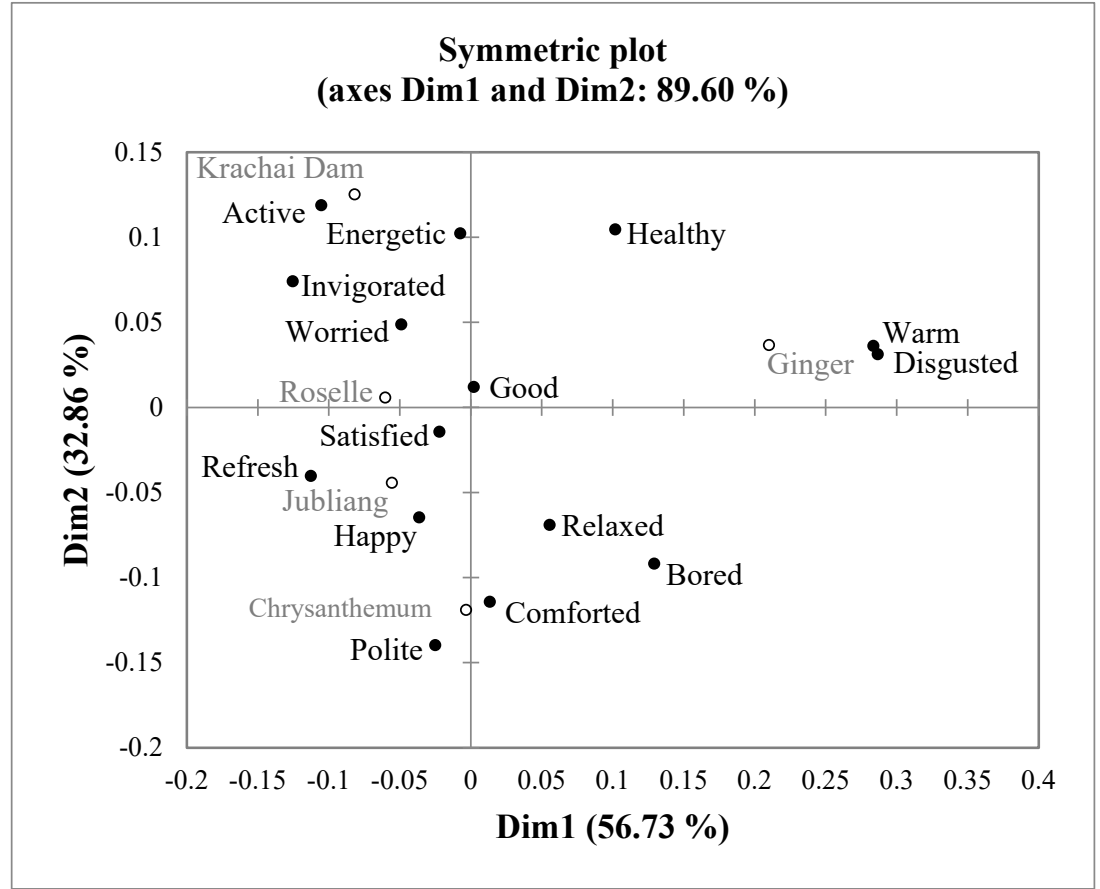

Figure 4. CA symmetric plots of emotion and wellness responses of the five herbal drinks in the (A) CATA-Words and (B) RATA-Words questionnaires. Symbols $(\bigcirc)$ and $(\bullet)$ indicate the position of herbal drinks and emotion and wellness terms, respectively.

Meyners et al. [27] mentioned that compared to CATA questions, the use of a RATA variant was found to increase the number of attribute terms selected to describe samples and led to a slight increase in the percentage of terms for which significant differences among samples were identified. Jaeger et al. [40] compared the CATA and RATA question formats using an emoji questionnaire. They found that neither CATA nor RATA emojiquestions were regarded by consumers as difficult or tedious. Their recommendation for 
method selection was to use CATA emoji questions when samples have distinct emotional associations, whereas RATA seems to better discriminate between samples with more similar emotional profiles. However, in this study, the CA configurations of CATA data vs. RATA data revealed only slightly different relationships between herbal drinks and emotion and wellness profiles (Figure 4).

\subsection{Overall Discussion}

Overall, all types of data (rating, RATA, and CATA) showed similar results in terms of emotion and wellness profiles of the five herbal drinks studied. The "warm" and "healthy" terms were important and relevant to ginger drink, and the negative emotion term "disgusted" was elicited. "Refreshed" and "invigorated" were the important wellness terms for roselle drink. "Active", "energetic", and "worried" emotion terms were often elicited by Krachai Dam drink. Additionally, "comforted", "relaxed", and "bored" were significant terms for describing the chrysanthemum and Jubliang drinks. Although chrysanthemum drink had the highest overall liking scores (Table 5), some consumers expressed a "bored" emotion. It is a challenge of product developers to reduce the negative emotions associated with products. Elicited negative emotion terms should be carefully considered when developing or improving products. In the case of herbal drinks, ginger drink was associated with "disgusted" by some consumers, possibly because of its natural spicy flavor. Krachai Dam drink was associated with "worried" because it is not well-known by many Thai consumers and it normally imparts a pungent and sour flavor. "Bored" was elicited by chrysanthemum, possibly because it is a well-known traditional product that has not changed for a long time (i.e., not much product innovation applied to this drink).

When the three types of measuring methods-a rating scale, CATA question, and RATA question-were compared, they all produced similar emotion and wellness profiles, though there were advantages and limitations for each method. For a rating scale, the participants needed more time to complete the questionnaire than for other methods. However, a rating scale is appropriate for measuring intensity of attributes. The PCA and CA of rating scores were able to differentiate five herbal drinks into four groups with a higher percentage explained variance $(78.98 \%)$ when compared with the RATA score (three groups; $77.50 \%$ ). The CATA questionnaire was the most consumer-friendly method and was found to be appropriate for use for measurement of the presence or absence of the selected attributes. DA of CATA data identified the important terms discriminating the overall differences among the herbal tested drinks with the highest percentage of explained variance (93.6\% total for Can1 and Can2). Additionally, CA of CATA data separated the five herbal drinks into four groups, similar to those of the rating data, with a higher percentage of explained variance $(94.79 \%$ ) when compared with the RATA frequency (four groups, $89.60 \%$ ). However, the RATA score and RATA frequency from the RATA-Words question seemed to show better discrimination ability in term of improving the number of significant terms among the five herbal drinks.

\section{Conclusions}

In this study, we demonstrated the effects of two factors in designing a questionnaire for measurement of emotion and wellness responses elicited by herbal drinks consumed by Thai consumers. The first factor was questionnaire items in the form of words vs. full sentences. Results indicate that measuring emotion and wellness using full sentences did not provide a clear benefit over using words alone. When using familiar terms clearly understood by consumers, a full sentence in the questionnaire is not needed. For the second factor, three types of measuring methods, including a rating scale, CATA question, and RATA question were compared for their ability to discriminate among five herbal drinks. All three measuring methods produced similar emotion and wellness profiles. However, each method has advantages and limitations that researchers should carefully consider. Overall, this study provides some useful options in terms of questionnaire-item formats and measurement methods in the design of questionnaire to measuring consumers' emotion and 
wellness responses to herbal drinks. The information elucidated in this study is applicable to other food products and beverages. In the future, the impact of health-benefit statements on consumer perception and purchase intent with regard to herbal drinks formulated in our lab will be assessed by product tasting and with an appropriate sample size representing the target population. We also plan to collaborate with a medical school for human clinical trials to determine the health benefits of these herbal drinks.

Author Contributions: Conceptualization, W.P. and A.S.-E.; methodology, W.P., A.S.-E. and P.H.; formal analysis, A.S.-E. and P.H.; investigation, A.S.-E. and P.H.; writing-original draft preparation, P.H.; writing-review and editing, A.S.-E. and W.P.; visualization, P.H.; supervision, A.S.-E. and W.P.; project administration, A.S.-E.; funding acquisition, A.S.-E. and P.H. All authors have read and agreed to the published version of the manuscript.

Funding: This research receives funding from Sakon Nakhon Rajabhat University (grant no. 0213).

Institutional Review Board Statement: The study was reviewed and approved by the Ethics Committee of Khon Kaen University (HE611230, approved on 7 June 2018).

Informed Consent Statement: Informed consent was obtained from all subjects involved in the study.

Data Availability Statement: The data presented in this study are available in the manuscript.

Acknowledgments: The authors express their grateful appreciation to Sakon Nakhon Rajabhat University for financial support. The department of Food Technology, Faculty of Technology, Khon Kaen University, Thailand and the School of Nutrition and Food Sciences, Louisiana State University, Agricultural Center, Baton Rouge, LA, USA, are also gratefully acknowledged for their support. We would also like to thank the consumers who participated in this research.

Conflicts of Interest: The authors declare no conflict of interest. The funders had no role in the design of the study; in the collection, analyses, or interpretation of data; in the writing of the manuscript, or in the decision to publish the results.

\section{References}

1. Spinelli, S.; Niedziela, M. Chapter 5 Emotion Measurements and Application to Product and Packaging Development. In Integrating the Packaging and Product Experience in Food and Beverages: A Road-Map to Consumer Satisfaction; Burgess, P., Ed.; Woodhead: Publishing, UK, 2016; pp. 77-119.

2. Jiang, Y.; King, J.M.; Prinyawiwatkul, W. A review of measurement and relationships between food, eating behavior and emotion. Trends Food Sci. Technol. 2014, 36, 15-28. [CrossRef]

3. Köster, E.P.; Mojet, J. From mood to food and from food to mood: A psychological perspective on the measurement of food-related emotions in consumer research. Food Res. Int. 2015, 76, 180-191. [CrossRef]

4. Volz, K.G.; Hertwig, R. Emotions and decisions: Beyond conceptual vagueness and the rationality muddle. Perspect. Psychol. Sci. 2016, 11, 101-116. [CrossRef] [PubMed]

5. King, S.C.; Meiselman, H.L.; Carr, B.T. Measuring emotions associated with foods in consumer testing. Food Qual. Prefer. 2010, 21, 1114-1116. [CrossRef]

6. Ng, M.; Chaya, C.; Hort, J. Beyond liking: Comparing the measurement of emotional response using EsSense Profile and consumer defined check-all-that-apply methodologies. Food Qual. Prefer. 2013, 28, 193-205. [CrossRef]

7. Spinelli, S.; Masi, C.; Dinnella, C.; Zoboli, G.P.; Monteleone, E. How does it make you feel? A new approach to measuring emotions in food product experience. Food Qual. Prefer. 2014, 37, 109-122. [CrossRef]

8. Schouteten, J.J.; Steur, H.D.; Pelsmaeker, S.D.; Lagast, S.; Bourdeaudhuij, I.D.; Gellynck, X. An integrated method for the emotional conceptualization and sensory characterization of food products: EmoSensory ${ }^{\circledR}$ Wheel. Food Res. Int. 2015, 78, 96-107. [CrossRef]

9. King, S.C.; Snow, J.; Meiselman, H.L.; Sainsbury, J.; Carr, B.T.; McCafferty, D.; Serrano, D.; Gillette, M.; Millard, L.; Li, Q. Development of a questionnaire to measure consumer wellness associated with foods: The WellSense Profile ${ }^{\mathrm{TM}}$. Food Qual. Prefer. 2015, 39, 82-94. [CrossRef]

10. Apaolaza, V.; Hartmann, P.; D’Souza, C.; López, M. Eat organic-Feel good? The relationship between organic food consumption, health concern and subjective wellbeing. Food Qual. Prefer. 2018, 63, 51-62. [CrossRef]

11. Hanmontree, P.; Sae-Eaw, A. Krachai Dam (Kaempferia parviflora) drinks: Physicochemical properties, consumer acceptance, purchase intent, and emotion and wellness responses. Ital. J. Food Sci. 2020, 32, 631-644.

12. Jang, J.A.; Oh, J.E.; Na, Y.; Yeo, G.E.; Cho, M.S. Emotions evoked by colors and health functionality information of colored rice: A cross-cultural study. Foods 2021, 10, 231. [CrossRef] [PubMed]

13. Hu, X.; Lee, J. Emotions elicited while drinking coffee: A cross-cultural comparison between Korean and Chinese consumers. Food Qual. Prefer. 2019, 76, 160-168. [CrossRef] 
14. Gunaratne, T.M.; Viejo, C.G.; Fuentes, S.; Torrico, D.D.; Gunaratne, N.M.; Ashman, H.; Dunshea, F.R. Development of emotion lexicons to describe chocolate using the Check-All- That-Apply (CATA) methodology across Asian and Western groups. Food Res. Int. 2019, 115, 526-534. [CrossRef] [PubMed]

15. Farzaneh, V.; Carvalho, I.S. A review of the health benefit potentials of herbal plant infusions and their mechanism of actions. Ind. Crops Prod. 2015, 65, 247-258. [CrossRef]

16. Craig, W.J. Health-promoting properties of common herbs. Am. J. Clin. Nutr. 1999, 70, 491s-499s. [CrossRef]

17. Jabeur, I.; Pereira, E.; Barros, L.; Calhelha, R.C.; Soković, M.; Oliveira, M.B.P.P.; Ferreira, I.C.F.R. Hibiscus sabdariffa L. as a source of nutrients, bioactive compounds and colouring agents. Food Res. Int. 2017, 100, 717-723. [CrossRef]

18. Da-Costa-Rocha, I.; Bonnlaender, B.; Sievers, H.; Pischel, I.; Heinrich, M. Hibiscus sabdariffa L.-A phytochemical and pharmacological review. Food Chem. 2014, 165, 424-443. [CrossRef]

19. Shi, X.-F.; Chu, J.-Z.; Zhang, Y.-F.; Liu, C.-Q.; Yao, X.-Q. Nutritional and active ingredients of medicinal chrysanthemum flower heads affected by different drying methods. Ind. Crops Prod. 2017, 104, 45-51. [CrossRef]

20. Pratap, S.R.; Gangadharappa, H.V.; Mruthunjaya, K. Ginger: A potential neutraceutical, an updated review. Int. J. Pharmacogn. Phytochem. Res. 2017, 9, 1227-1238. [CrossRef]

21. Kruawan, K.; Kangsadalampai, K. Antioxidant activity, phenolic compound contents and antimutagenic activity of some water extract of herbs. Thai J. Pharm. Sci. 2006, 30, 28-35.

22. Chen, D.; Li, H.; Li, W.; Feng, S.; Deng, D. Kaempferia parviflora and Its Methoxyflavones: Chemistry and Biological Activities. Evid.-Based Complement. Alternat. Med. 2018, 2018, 4057456. [CrossRef] [PubMed]

23. Poonnakasem, N.; Pujols, K.D.; Chaiwanichsiri, S.; Laohasongkram, K.; Prinyawiwatkul, W. Different oils and health benefit statements affect physicochemical properties, consumer liking, emotion, and purchase intent: A case of sponge cake. J. Food Sci. 2016, 81, S165-S173. [CrossRef] [PubMed]

24. Wardy, W.; Jack, A.R.; Chonpracha, P.; Alonso, J.R.; King, J.M.; Prinyawiwatkul, W. Gluten-free muffins: Effects of sugar reduction and health benefit information on consumer liking, emotion, and purchase intent. Int. J. Food Sci. Technol. 2018, 53, 262-269. [CrossRef]

25. Meilgaard, M.C.; Civille, G.V.; Carr, B.T. Sensory Evaluation Techniques, 5th ed.; CRC Press: Boca Raton, FL, USA, 2016; p. 589.

26. Ares, G.; Bruzzone, F.; Vidal, L.; Cadena, R.S.; Giménez, A.; Pineau, B.; Hunter, D.C.; Paisley, A.G.; Jaeger, S.R. Evaluation of a rating-based variant of check-all-that-apply questions: Rate-all-that-apply (RATA). Food Qual. Prefer. 2014, 36, 87-95. [CrossRef]

27. Meyners, M.; Jaeger, S.R.; Ares, G. On the analysis of Rate-All-That-Apply (RATA) data. Food Qual. Prefer. 2016, 49, 1-10. [CrossRef]

28. Ngoenchai, P.; Alonso, J.R.; Suwonsichon, T.; Suwonsichon, S.; Prinyawiwatkul, W. Effects of visual cues on consumer expectation, emotion and wellness responses, and purchase intent of red chili powders. J. Food Sci. 2019, 84, 3018-3026. [CrossRef]

29. King, S.C.; Meiselman, H.L. Development of a method to measure consumer emotions associated with foods. Food Qual. Prefer. 2010, 21, 168-177. [CrossRef]

30. Cardello, A.V.; Meiselman, H.L.; Schutz, H.G.; Craig, C.; Given, Z.; Lesher, L.L.; Eicher, S. Measuring emotional responses to foods and food names using questionnaires. Food Qual. Prefer. 2012, 24, 243-250. [CrossRef]

31. King, S.C.; Meiselman, H.L.; Carr, B.T. Measuring emotions associated with foods: Important elements of questionnaire and test design. Food Qual. Prefer. 2013, 28, 8-16. [CrossRef]

32. Calvo-Porral, C.; Rivaroli, S.; Orosa-González, J. The asymmetric emotional associations to beverages: An approach through the theory of positive asymmetry. Foods 2021, 10, 794. [CrossRef]

33. Soares, E.K.B.; Esmerino, E.A.; Ferreira, M.V.S.; da Silva, M.A.A.P.; Freitas, M.Q.; Cruz, A.G. What are the cultural effects on consumers' perceptions? A case study covering coalho cheese in the Brazilian northeast and southeast area using word association Food Res. Int. 2017, 102, 553-558. [CrossRef] [PubMed]

34. Cao, X.; Xiong, X.; Xu, Z.; Zeng, Q.; He, S.; Yuan, Y.; Wang, Y.; Yang, X.; Su, D. Comparison of phenolic substances and antioxidant activities in different varieties of chrysanthemum flower under simulated tea making conditions. J. Food Meas. Charact. 2020, 14, 1443-1450. [CrossRef]

35. Chen, S.; Liu, J.; Dong, G.; Zhang, X.; Liu, Y.; Sun, W.; Liu, A. Flavonoids and caffeoylquinic acids in Chrysanthemum morifolium Ramat flowers: A potentially rich source of bioactive compounds. Food Chem. 2021, 344, 128733. [CrossRef] [PubMed]

36. Ghafoor, K.; Juhaimi, F.A.; Özcan, M.M.; Uslu, N.; Babiker, E.E.; Ahmed, I.A.M. Total phenolics, total carotenoids, individual phenolics and antioxidant activity of ginger (Zingiber officinale) rhizome as affected by drying methods. Food Sci. Technol. 2020, 126, 109354. [CrossRef]

37. Nguyen, Q.V.; Chuyen, H.V. Processing of herbal tea from roselle (Hibiscus sabdariffa L.): Effects of drying temperature and brewing conditions on total soluble solid, phenolic content, antioxidant capacity and sensory quality. Beverages 2020, 6, 2. [CrossRef]

38. Riaz, G.; Chopra, R. A review on phytochemistry and therapeutic uses of Hibiscus sabdariffa L. Biomed. Pharmacother. 2018, 102, 575-586. [CrossRef]

39. Olegario, L.S.; Estevéz, M.; González-Mohino, A.; Madruga, M.S.; Ventanas, S. Cross-cultural emotional response to food stimuli: Influence of consumption context. Food Res. Int. 2021, 142, 110194. [CrossRef]

40. Jaeger, S.R.; Lee, S.M.; Kim, K.-O.; Chheang, S.L.; Roigard, C.M.; Ares, G. CATA and RATA questions for product-focused emotion research: Five case studies using emoji questionnaires. Food Qual. Prefer. 2018, 68, 342-348. [CrossRef] 